\title{
ON REJECTION TO INFINITY AND EXTERIOR MOTION IN THE RESTRICTED PROBLEM OF THREE BODIES*
}

\author{
BY \\ BERNARD OSGOOD KOOPMAN
}

INTRODUCTION

The cases of the restricted problem of three bodies which have hitherto been considered in detail deal with motion of the particle in the interior of a closed oval of zero velocity, or in the neighborhood of points of equilibrium or periodic orbits. In none of these cases does the question of rejection of the infinitesimal body to infinity play any rôle. It is to the investigation of this phenomenon, and of types of motion in which its possibility is an essential feature, that the present paper is devoted. Attention is restricted to the case of motion in a plane.

Chapter I consists of a qualitative study of the individual half orbits which are described by the particle as it goes to or comes from infinity. The methods are altogether elementary. The first salient result is the proof of the existence of such half orbits, with a practical criterion for finding them. The second is the theorem that every half orbit which extends to infinity resembles a hyperbolic or parabolic orbit in this: that it has an asymptote (in the fixed space), and the particle has a limiting velocity. In a special case, resembling the parabolic, this velocity is zero and the asymptote is at infinity (projectively speaking).

In Chapter II, we take up the investigation of infinity from the point of view of a singularity of the differential equations of motion. It is shown that by means of a rational substitution this singularity can be reduced to a well known type: a point, at which the right-hand members of a first-order system are analytic, but vanish simultaneously. The power expansions of these functions about the point start with terms of the third degree. When all further terms are omitted, a system is obtained whose solution is wholly known; unfortunately, no method is at hand for extending its properties to the given system. We finish the chapter with a discussion of the totality of half orbits which reach to infinity, regarded as an ensemble.

* Presented to the Society, January 2, 1926; received by the editors in April, 1926. In substance this paper is the author's Doctor's thesis, presented at Harvard, 1925. 
It is known that for sufficiently large values of the constant of Jacobi there is a closed oval of zero velocity containing the two massive bodies; its exterior is a possible region of motion of the particle. This constitutes the simplest case in which rejection to infinity can occur, and forms the subject of the last two chapters.

Chapter III deals with the surface of section, a concept which has become familiar through the writings of Poincare and Birkhoff. We show that our dynamical problem possesses a ring-shaped surface of section, bounded by a periodic orbit, and by the singularity at infinity. We employ the familiar method of generalizing by continuity from an integrable case. Our results are valid only for sufficiently small values of the ratio of the masses of the heavy bodies.

In Chapter IV, we begin by extending to our case the results concerning the existence and properties of symmetric periodic orbits which were obtained by Birkhoff in the case of motion inside an oval of zero velocity. Then we consider the properties and distribution of the complete orbits which reach to infinity in one or in both directions. As everything here is a consequence of the transformation on the surface of section, it is subject to the same restrictions under which the existence of the latter has been established.

Chapter I. ON orbits EXtending to INFinity

1. The equations of motion. The restricted problem of three bodies in the plane can, by a proper choice of units of mass, length, and time, be reduced to the study of the motion of a particle $P$ under the Newtonian attraction of two bodies $S$ and $J$, of masses $\mu$ and $1-\mu$, which revolve in circles about their mutual center of gravity $O$ at unit angular velocity. The distances $\overline{O S}$ and $\overline{O J}$ will then be $1-\mu$ and $\mu$ respectively.

Let the fixed $X Y$-axes be chosen through the center of gravity of $S$ and $J$, in the plane of motion, and in such a manner that $S$ and $J$ revolve in the positive direction (Fig. 1). The equations of motion of $P:(X, Y)$ will be

$$
\begin{gathered}
\frac{d^{2} X}{d t^{2}}=-\frac{\partial \Pi}{\partial X}, \quad \frac{d^{2} Y}{d t^{2}}=-\frac{\partial \Pi}{\partial Y}, \\
\Pi=\Pi(X, Y, t)=-\frac{1-\mu}{r_{1}}-\frac{\mu}{r_{2}} .
\end{gathered}
$$

In polar coördinates, $P:(r, \theta)$ :

$$
\frac{d^{2} r}{d t^{2}}-r\left(\frac{d \theta}{d t}\right)^{2}=-\frac{\partial \Pi}{\partial r}, \quad \frac{d}{d t}\left(r^{2} \frac{d \theta}{d t}\right)=-\frac{\partial \Pi}{\partial \theta} .
$$


If the rotating $x y$-axes are introduced (Fig. 1), with respect to which $S$ and $J$ are fixed and of zero ordinate, the equations may be written in the well known Jacobi form:

$$
\begin{aligned}
& \frac{d^{2} x}{d t^{2}}-2 \frac{d y}{d t}=\frac{\partial \Omega}{\partial x}, \quad \frac{d^{2} y}{d t^{2}}+2 \frac{d x}{d t}=\frac{\partial \Omega}{\partial y}, \\
& \Omega=\Omega(x, y)=\frac{1}{2} r^{2}-\Pi=\frac{1}{2} r^{2}+\frac{1-\mu}{r_{1}}+\frac{\mu}{r_{2}}, \\
& r_{1}=\left[(x-\mu)^{2}+y^{2}\right]^{1 / 2}, \quad r^{2}=\left[(x+1-\mu)^{2}+y^{2}\right]^{1 / 2}, \\
& r=\left(x^{2}+y^{2}\right)^{1 / 2} .
\end{aligned}
$$

They admit the integral of Jacobi

$$
v^{2}=\left(\frac{d x}{d t}\right)^{2}+\left(\frac{d y}{d t}\right)^{2}=2 \Omega-C .
$$

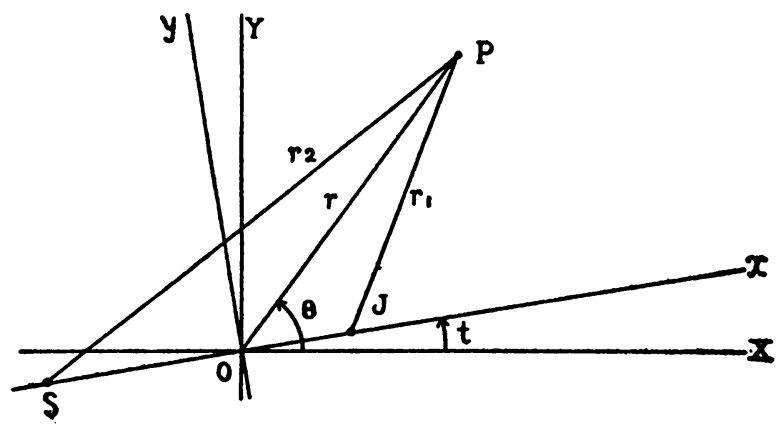

Fig. 1

Our problem does not in general possess an integral of energy; hence we cannot speak of the "energy of the system." It will, however, be useful to introduce the quantity $\eta$, which will be called the specific energy of the particle $P$ :

$$
\begin{aligned}
\eta & =\frac{1}{2} V^{2}+\Pi \\
& =\frac{1}{2}\left[\left(\frac{d x}{d t}-y\right)^{2}+\left(\frac{d y}{d t}+x\right)^{2}\right]+\Pi, \\
V^{2} & =\left(\frac{d X}{d t}\right)^{2}+\left(\frac{d Y}{d t}\right)^{2} .
\end{aligned}
$$

During a given motion, $\eta$ evidently changes with the time. Its physical interpretation is $\eta=E / \epsilon$, where $E$ is the total energy that $P$ would have if its mass were $\epsilon$ and if $S$ and $J$ were fixed at the positions they occupy at the time $t$ in question. 
If we introduce the double areal velocity $G$ :

$$
G=r^{2} \frac{d \theta}{d t},
$$

the integral of Jacobi may be written in the form

$$
2(G-\eta)=C .
$$

2. Some theorems on stability.* When $\mu=0$, we have the problem of two bodies. Then $\eta$ remains constant as $P$ moves along a given orbit, for the system admits the integral of energy. Its value has a fundamental bearing on the stability of the motion, a fact which follows from the familiar theorem of celestial mechanics The orbit is hyperbolic, parabolic, or elliptic, according as $\eta>0, \eta=0$, or $\eta<0$. (This will be seen, e.g., from the equation $2 \eta G^{2}=e^{2}-1$ proved on page 313.) The theorems of this section may be regarded as the generalization of this fact to $\mu>0$.

Theorem 1. As long as $\eta \leqq \eta_{0}<0, P$ will lie within the rotating equipotential oval $\Pi=\eta_{0}$, and hence within a fixed distance of the origin which depends uniquely on the constant $\eta_{0}$.

For, from (4), $\Pi \leqq \eta \leqq \eta_{0}$. As $r_{1}$ increases from 0 to $+\infty, \Pi$ increases from $-\infty$ to 0 . Hence $P$ lies within the oval $\Pi=\eta_{0}$. The last part of the theorem follows from the known form of the equipotentials about a pair of Newtonian particles.

We shall now consider one of the halves $A b$ into which an orbit is divided by a point $A$ upon it (Fig. 2). The sense in which the particle $P$ describes $A b$ does not matter. We orient $A b$ in the sense $A b$, and denote by $s$ the arclength measured from $A$. Likewise, we take the orientations $O X$ and $O P$. The angles $\theta$ and $\psi$ are measured positively from $O X$ to $O P$, and from $O P$ to $A B$, respectively; and $\phi=\theta+\psi$. Let quantities pertaining to $A$ be indicated by subscripts $\left({ }_{0}\right)$, those pertaining to $P, P_{n}$, etc., by similarly marked letters. Finally let $0 \leqq \theta_{0}<2 \pi$.

THEOREM 2. On the hypothesis that

(a) $r_{0} \geqq 5 ;\left(>\left(5+17^{1 / 2}\right) / 2\right)$;

(b) either $0 \leqq \psi_{0} \leqq \pi / 2$, or else $3 \pi / 2 \leqq \psi_{0}<2 \pi$;

(c) the specific energy $\eta$ of the particle $P$ of which $A b$ is the orbit is positive or zero at every point of this curve; we conclude that

$(\alpha) A b$ extends to infinity without apsides (i.e., $\psi \neq \pi / 2$ or $3 \pi / 2$ );

\footnotetext{
* Cf. the note at the end of this section.
} 
(B) $\lim _{\mathrm{\theta} \rightarrow \infty} \theta$ exists $(=\Theta)$;

(r) $\lim _{8 \rightarrow \infty} \psi=0$;

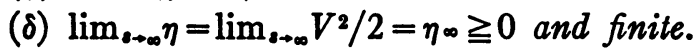

In our proof we shall first examine the analytic nature of the curve $A b$ and the quantities $r, \theta, s, \phi, \psi$. This is necessary in order to justify the use of many of the formulas of polar coördinates, etc. Then we shall establish some inequalities, (5), (6), (6'), of great importance in this and later theorems. Finally, we shall prove successively the conclusions $(\beta),(\gamma),(\alpha),(\delta)$.

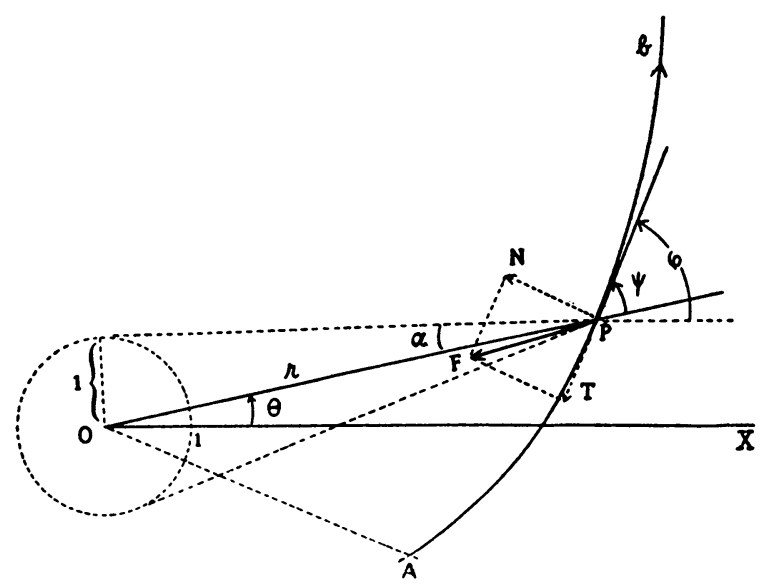

Frg. 2

Let us denote by $\{A b\}$ the connected portion of $A b$ which starts at $A$ and remains outside the circle $r=5$. It follows from well known existence theorems applied to the equations (1) that $\{A B\}$ may be represented as

$$
X=F_{1}(t), \quad Y=F_{2}(t),
$$

where $F_{1}(t)$ and $F_{2}(t)$ are analytic and single-valued for $t$ real and $t_{1} \leqq t \leqq t_{2}$ ( $t_{1}$ may be the symbol $-\infty$, or $t_{2}$ the symbol $+\infty$, - the equality sign, naturally, being omitted). Hence $\{A b\}$ may be represented as

$$
r=f_{1}(t), \quad \theta=f_{2}(t),
$$

and $f_{1}(t), f_{2}(t)$ will be of the same nature as $F_{1}(t), F_{2}(t)$. From hyp. (c) and formula (4) it follows that $d s / d t(=V)$ cannot vanish at a finite distance from the origin. Hence, $\{A b\}$ is an analytic curve without cusps. 
The arc, $s=s(t)$, is clearly analytic in $t$ for the values considered. Since $d s / d t \neq 0, s$ may be introduced as parameter, when we shall have

$$
r=r(s), \quad \theta=\theta(s), \quad \psi=\psi(s), \quad \phi=\theta+\psi=\phi(s),
$$

all these functions being single-valued and analytic for all real $s$ in the interval $0 \leqq s<s_{1}\left(s_{1}\right.$ may be the symbol $\left.+\infty\right)$. The same may be said of the curvature $\kappa=|d \phi / d s|$, except where $\kappa=0$, when this function is merely continuous.

We now turn to the inequalities. Let $F$ be the absolute value of the resultant $P F$ of the forces acting on $P$, and $N$ the absolute value of its normal component to the path, $T$ the algebraic value of its tangential component. Since $S$ and $J$ are always within the unit circle of center $O$, we have

$$
F \leqq \frac{1-\mu}{r_{1}^{2}}+\frac{\mu}{r_{2}^{2}} \leqq \frac{1-\mu}{(r-1)^{2}}+\frac{\mu}{(r-1)^{2}}=\frac{1}{(r-1)^{2}} .
$$

Further, as regards the line of action of the resultant, the angle $F P N$ lies between $\pi / 2-\psi-\alpha$ and $\pi / 2-\psi+\alpha$, where $\alpha=\sin ^{-1}(1 / r)$ is the angle between the radius-vector and one of the tangents through $P$ to the unit circle. ( $\angle F P N$ and $\alpha$ are "small" unoriented angles.) It follows that

$$
\begin{aligned}
N & =F|\cos (\angle F P N)|=F|\sin (\psi+\delta)|, \quad|\delta|<\alpha, \\
& =F|\sin \psi \cos \delta+\cos \psi \sin \delta| ;
\end{aligned}
$$

therefore

$$
\begin{aligned}
N & \leqq F(|\sin \psi| \cdot 1+|\cos \psi| \cdot \sin \alpha) \\
& \leqq F\left(|\sin \psi|+|\cos \psi| \cdot \frac{1}{r}\right) .
\end{aligned}
$$

Hence, from the previous inequality,

$$
N \leqq \frac{|\sin \psi|}{(r-1)^{2}}+\frac{|\cos \psi|}{r(r-1)^{2}} .
$$

The normal resolution of force and acceleration gives, by elementary mechanics,

$$
\kappa V^{2}=N \text {, }
$$

therefore

$$
\kappa V^{2} \leqq \frac{|\sin \psi|}{(r-1)^{2}}+\frac{|\cos \psi|}{r(r-1)^{2}}
$$


By hyp. (c),

therefore

$$
\eta=\frac{V^{2}}{2}+\Pi \geqq 0
$$

$$
V^{2} \geqq-2 \Pi \text {. }
$$

Considerations like those of the preceding paragraph lead to the inequality

$$
-2 \Pi=2\left(\frac{1+\mu}{r_{1}}+\frac{\mu}{r_{2}}\right) \geqq \frac{2}{r+1},
$$

therefore

whence

$$
V^{2} \geqq \frac{2}{r+1}
$$

$$
\kappa \leqq \frac{r+1}{2(r-1)^{2}}|\sin \psi|+\frac{r+1}{2 r(r-1)^{2}}|\cos \psi|,
$$

which is the first of the inequalities we are seeking.

A first result of this inequality is that the curvature of $\{A b\}$ at an apsis $\left(r_{1}, \theta_{1}\right)$ is less than the curvature of the circle of radius $r_{1}$ (not $r_{1}$ of Fig. 1). For, by (5), when $\psi=\pi / 2$,

$$
\kappa_{1} \leqq \frac{r_{1}+1}{2\left(r_{1}-1\right)^{2}}=\frac{1}{r_{1}} \frac{r_{1}\left(r_{1}+1\right)}{2\left(r_{1}-1\right)^{2}},
$$

and a little computation shows that when $r \geqq 5$,

therefore

$$
\frac{r_{1}\left(r_{1}+1\right)}{2\left(r_{1}-1\right)^{2}}<1
$$

$$
\kappa_{1}<\frac{1}{r_{1}} .
$$

It follows that $r(s)$ can have no maxima, i.e., $\{A b\}$ has no higher apsides. This fact, which is almost geometrically evident from the last inequality, is proved by considering the osculating circle $C$ drawn at a point $\left(r_{1}, \theta_{1}\right)$ where we suppose $d r / d s=0$. It is tangent externally to the circle $K$ of radius $\boldsymbol{r}_{1}$, center at $O$. Since $\{A b\}$ has contact of higher order with $C$, it will lie outside $K$ in the neighborhood of $\left(r_{1}, \theta_{1}\right)$. Hence $\left(r_{1}, \theta_{1}\right)$ is not a point of maximum $r$. It follows from this, and from hyp. (a), (b), that $r(s)$ increases monotonically with $s$. Hence $\{A b\}$ is identical with $A b$, and all our above results, obtained for the former curve, apply to the whole half orbit. 
The length of $A b$ is infinite. For if $A b$ were within a fixed distance of the origin, there would exist a constant, $h>0$, such that $|d s / d t|>h$ (by (4) and hyp. (c)). Hence the length of $A b$ would be infinite. And of course it would be infinite if the contrary assumption were made about $A b$.

We return to the inequality (5). Recalling the elementary formulas of polar coördinates:

$$
\sin \psi=r \frac{d \theta}{d s}, \quad \cos \psi=\frac{d r}{d s},
$$

which are valid through $A b$, by the nature of this curve, we have

$$
\left|\frac{d \phi}{d s}\right|=\kappa \leqq \frac{r+1}{2(r-1)^{2}}|\sin \psi|+\frac{r+1}{2 r(r-1)^{2}}|\cos \psi|,
$$

therefore

$$
|d \phi| \leqq r \frac{(r+1)}{2(r-1)^{2}}|d \theta|+\frac{r+1}{2 r(r-1)^{2}}|d r| .
$$

We shall take, as we may, $d s>0$. Because of the monotonic increase of $r, d r \geqq 0$, and $|\psi| \leqq \pi / 2$. Consider first the case where $d \theta \geqq 0$ between two points $\left(r_{1}, \theta_{1}\right)$ and $\left(r_{2}, \theta_{2}\right), r_{1} \leqq r_{2}$. We are lead to

$$
\begin{aligned}
& d \phi \leqq|d \phi|<\frac{r(r+1)}{2(r-1)^{2}} d \theta+\frac{r+1}{2 r(r-1)^{2}} d r \\
& d \psi=d \phi-d \theta<\left[\frac{r(r+1)}{2(r-1)^{2}}-1\right] d \theta+\frac{r+1}{2 r(r-1)^{2}} d r .
\end{aligned}
$$

A little computation shows that the bracket decreases as $r$ increases when $r \geqq 5$, so that

$$
\frac{r(r+1)}{2(r-1)^{2}}-1 \leqq-\frac{1}{16}, \quad \text { when } r \geqq 5
$$

Hence

$$
d \psi \leqq-\frac{1}{16} d \theta+\frac{r+1}{2 r(r-1)^{2}} d r .
$$

Taking the definite integral of this along $A b$ from $\left(r_{1}, \theta_{1}\right)$ to $\left(r_{2}, \theta_{2}\right)$,

$$
\psi_{2}-\psi_{1} \leqq-\frac{1}{16}\left(\theta_{2}-\theta_{1}\right)+\left\{\frac{1}{r_{1}-1}-\frac{1}{r_{2}-1}+\frac{1}{2} \log \left(\frac{r_{2}}{r_{1}}\right) \frac{r_{1}-1}{r_{2}-1}\right\} ;
$$


since $r_{2} \geqq r_{1} \geqq 5$, this yields

$$
\psi_{2}-\psi_{1} \leqq-\frac{1}{16}\left(\theta_{2}-\theta_{1}\right)+\frac{1}{r_{1}-1}-\frac{1}{r_{2}-1} .
$$

Turn now to the case where $d \theta \leqq 0$ between $\left(r_{1}, \theta_{1}\right)$ and $\left(r_{2}, \theta_{2}\right)$. In the work up to now we have made no use of the fact that $S$ and $J$ are revolving about $O$ in the "counterclockwise" direction. There is, then, nothing to distinguish the formulas we have obtained from those we should have found had we measured all angles positively in the "clockwise" sense. Accordingly, our last result (6) must hold when $\theta, \phi, \psi$ are replaced by their negatives, and the condition $d \theta \geqq 0$ by $d(-\theta) \geqq 0$, i.e., $d \theta \leqq 0$. Hence, after multiplying through by -1 , we get

$$
\psi_{2}-\psi_{1} \geqq-\frac{1}{16}\left(\theta_{2}-\theta_{1}\right)-\frac{1}{r_{1}-1}+\frac{1}{r_{2}-1} .
$$

This formula replaces (6) when $\theta$ decreases monotonically between $\left(r_{1}, \theta_{1}\right)$ and $\left(r_{2}, \theta_{2}\right)$.

We are now in a position to prove the conclusion $(\beta)$. Assume first that from a certain point $\left(r_{1}, \theta_{1}\right)$ on, $\theta$ increases monotonically with $s$. From (6) we have, since $r_{1} \geqq 5$,

$$
\psi_{2}-\psi_{1} \leqq-\frac{1}{16}\left(\theta_{2}-\theta_{1}\right)+\frac{1}{4}
$$

Now $0 \leqq \psi_{1} \leqq \pi / 2,0 \leqq \psi_{2} \leqq \pi / 2$, and hence

therefore

$$
-\frac{\pi}{2} \leqq-\frac{1}{16}\left(\theta_{2}-\theta_{1}\right)+\frac{1}{4} ;
$$

$$
\theta_{2} \leqq \theta_{1}+4+8 \pi \text {. }
$$

Thus, $\theta_{2}$ (or $\theta$ ) increases steadily but remains finite as $s_{2} \rightarrow \infty$. Hence it approaches a limit, $\Theta$, as we wished to prove. The same conclusion is arrived at when $\theta$ decreases monotonically.

The only case where $\theta$ does not behave as we have assumed is when it passes through infinitely many maxima and minima as $s \rightarrow \infty$. Let these occur at $P_{2 k}$ and $P_{2 k-1}$ respectively $(k=1,2, \cdots)$. At these points the angle $\psi$ will be zero. Hence, by $(6),\left(6^{\prime}\right)$,

$$
\begin{aligned}
& \left|\theta_{2 k}-\theta_{2 k-1}\right| \leqq 16\left(\frac{1}{r_{2 k-1}-1}-\frac{1}{r_{2 k}-1}\right), \\
& \left|\theta_{2 k+1}-\theta_{2 k}\right| \leqq 16\left(\frac{1}{r_{2 k}-1}-\frac{1}{r_{2 k+1}-1}\right) .
\end{aligned}
$$


Let $P^{\prime}$ and $P^{\prime \prime}$ be an arbitrary pair of points on $A b, s^{\prime}<s^{\prime \prime}$. If $\theta^{\prime}<\theta^{\prime \prime}$, call $P_{2 p-1}$ the minimum adjacent to (or coincident with) $P^{\prime}$, and $P_{2 q}$ the maximum adjacent to (or coincident with) $P^{\prime \prime}$. If $\theta^{\prime}>\theta^{\prime \prime}$, call $P_{2 p}$ the maximum adjacent to $P^{\prime}$, and $P_{2 q-1}$ the minimum adjacent to $P^{\prime \prime}$. It will be seen that as $s^{\prime}$ and $s^{\prime \prime}$ become infinite, the $s$ for the other corresponding points do likewise. Also, $p \leqq q$. In the first case, $\left(\theta^{\prime}<\theta^{\prime \prime}\right)$, we have

$$
\begin{aligned}
\left|\theta^{\prime \prime}-\theta^{\prime}\right| & \leqq\left|\theta_{2 q}-\theta_{2 p-1}\right| \leqq\left|\theta_{2 p}-\theta_{2 p-1}\right|+\left|\theta_{2 p+1}-\theta_{2 p}\right|+\cdots+\left|\theta_{2 q}-\theta_{2 q-1}\right| \\
& \leqq 16\left\{\frac{1}{r_{2 p-1}-1}-\frac{1}{r_{2 p}-1}+\frac{1}{r_{2 p}-1}-\frac{1}{r_{2 p+1}-1}+\cdots-\frac{1}{r_{2 q}-1}\right\},
\end{aligned}
$$

therefore

$$
\left|\theta^{\prime \prime}-\theta^{\prime}\right| \leqq 16\left\{\frac{1}{r_{2 p-1}-1}-\frac{1}{r_{2 q}-1}\right\} .
$$

Similarly, in the case $\theta^{\prime}>\theta^{\prime \prime}$,

$$
\left|\theta^{\prime \prime}-\theta^{\prime}\right| \leqq 16\left\{\frac{1}{r_{2 p}-1}-\frac{1}{r_{2 q-1}-1}\right\} .
$$

We have seen that $r$ increases monotonically with $s$. Hence, as $s \rightarrow \infty$, either $r \rightarrow \infty$, or else $\lim r=R \geqq 5$. In either case the braces in the last two inequalities approach zero. Thus

$$
\lim \left|\theta^{\prime \prime}-\theta^{\prime}\right|=0
$$

as $s^{\prime}$ and $s^{\prime \prime}$ become infinite independently of one another. It follows from a fundamental theorem of analysis that

$$
\lim _{s \rightarrow \infty} \theta=\Theta,
$$

and thus conclusion $(\beta)$ is established in all cases.

Turn now to the conclusion $(\gamma)$ or our theorem. When $\theta$ changes monotonically with $s$ from a certain point on, it follows from (6) or $\left(6^{\prime}\right)$ that $\lim \left|\psi^{\prime \prime}-\psi^{\prime}\right|=0$ as $s^{\prime}$ and $s^{\prime \prime}$ both become infinite; hence, $\lim _{s \rightarrow \infty} \psi$ exists. Assume it distinct from zero. By the formula $r d \theta / d r=\tan \psi$ it follows that

$$
\left|r \frac{d \theta}{d r}\right|>a>0
$$

from a certain point on; therefore

$$
|\theta|>a \log r+b \quad \text { ( } a, b \text { const. }) .
$$


If $r \rightarrow \infty$ with $s$, we are led to a contradition with $(\beta)$. If $r$ does not behave in this way, $\lim _{s \rightarrow \infty} r=R-$, and the formula $r d \theta / d s=\sin \psi$ leads to

$$
\left|\frac{d \theta}{d s}\right|=\frac{|\sin \psi|}{R}>A>0
$$

from a certain point on; therefore

$$
|\theta|>A s+B \quad(A, B \text { const. }) \text {. }
$$

Again we are brought into conflict with $(\beta)$. Thus, $(\gamma)$ is proved under the assumption of the monotonic change of $\theta$.

If $\theta$ is not monotonic, it will have an infinite succession of maxima and minima, at the points $P_{9 k}$ and $P_{2 k-1}$ respectively. Between $P_{2 k-1}$ and $P_{2 k}$, $\psi \geqq 0$; let $\psi^{(2 k)}$ be its maximum maximorum there, attained at the point $P^{(2 k)}: s_{2 k-1} \leqq s^{(2 k)} \leqq s_{2 k}$. By $(6)$,

$$
(0 \leqq) \psi^{(2 k)} \leqq-\frac{1}{16}\left(\theta^{(2 k)}-\theta_{2 k-1}\right)+\frac{1}{r_{2 k-1}-1}-\frac{1}{r^{(2 k)}-1} .
$$

Similarly, for the minimum minimorum $\psi^{(2 k+1)}$ between $P_{2 k}$ and $P_{2 k+1}$, we have

$$
(0 \geqq) \psi^{(2 k+1)} \geqq-\frac{1}{16}\left(\theta^{(2 k+1)}-\theta_{2 k}\right)-\frac{1}{r_{2 k}-1}+\frac{1}{r^{(2 k+1)}-1} .
$$

Hence it is readily seen that

$$
\lim _{k \rightarrow \infty} \psi^{(2 k)}=\lim _{k \rightarrow \infty} \psi^{(2 k+1)}=0,
$$

and the truth of the conclusion $(\gamma)$ is evident.

The proof of $(\alpha)$ now follows from the formula $d r / d s=\cos \psi$. For we see from $(\gamma)$ that

$$
\frac{d r}{d s}>M>0
$$

from a certain point on; therefore

$$
r>M s+N \quad(M, N \text { const. }),
$$

which makes $(\alpha)$ evident.

Conclusion ( $\delta$ ) follows from the formula of elementary mechanics giving the tangential resolution of force and acceleration of $P$ (Fig. 2):

$$
\frac{d V}{d t}=\frac{d}{d s}\left(\frac{V^{2}}{2}\right)=T \text {. }
$$


It is clear from what we have proved regarding the geometric nature of $A b$ that, from a certain point on, $T<0$. Hence $V^{2}$ decreases monotonically as $s \rightarrow \infty$. This renders the truth of $(\delta)$ obvious, and thus the proof of Theorem 2 is complete.

It is interesting to state these results in terms of the osculating eccentricity, $e$. Theorem 1 shows that as long as $e \leqq 1-h, h$ an arbitrarily small positive constant, the orbit remains within a fixed distance of the origin,* while in Theorem 2, we may replace the hyp. (c) by the requirement that $e \geqq 1$ along $A b$. We do not go into further detail, because there is a slight variance in usage in the definition of $e$. Once its meaning is fixed upon, the connection may be made with the value of $\eta$ by means of formulas analogous to the one cited in the opening paragraph of this section.

Theorem 2 cannot be applied directly to the testing of the stability of a half orbit given by its initial conditions, because it requires a knowledge concerning the value of $\eta$ throughout the entire length. But it is easy to derive a test which is directly applicable:

THEOREM 3. The conclusion of Theorem 2 subsists when the hypothesis (c) is replaced by the requirement that, at $A$,

$$
\left(\frac{d r}{d t}\right)_{0}^{2} \geqq \frac{2}{r_{0}-1}
$$

From hyp. (c) it follows that a segment $A B$ of $A b$ exists within which $|d r / d t|>0$. Letting $(r, \theta)$ denote any point on $A B$, we clearly have

$$
\frac{d^{2} r}{d t^{2}}=\frac{d r}{d t} \frac{d}{d r}\left(\frac{d r}{d t}\right)=\frac{1}{2} \frac{d}{d r}\left(\frac{d r}{d t}\right)^{2}
$$

By $\left(1^{\prime}\right)$, and Fig. 2,

$$
\frac{d^{2} r}{d t^{2}}=r\left(\frac{d \theta}{d t}\right)^{2}-\frac{\partial \Pi}{\partial r} \geqq-[\text { greatest possible } F] \geqq \frac{-1}{(r-1)^{2}} ;
$$

therefore

$$
\frac{d}{d r}\left(\frac{d r}{d t}\right)^{2} \geqq \frac{-2}{(r-1)^{2}} .
$$

Integrating from $A:\left(r_{0}, \theta_{0}\right)$ to $B:\left(r_{1}, \theta_{1}\right)$, with $r$ as independent variable, $d r>0$, we obtain axis.

- This is related to the classical theorem of celestial mechanics about the stability of the major 


$$
\left(\frac{d r}{d t}\right)_{1}^{2}-\frac{2}{r_{1}-1} \geqq\left(\frac{d r}{d t}\right)_{0}^{2}-\frac{2}{r_{0}-1} \geqq 0,
$$

the second inequality coming from hyp. $\left(c^{\prime}\right)$.

It follows that throughout $A b,|d r / d t|>0$. Otherwise, on letting $B$ approach the first point where $d r / d t=0$, our preceding formula would lead to a discontinuity in the function $d r / d t$, contrary to the nature which the functions $r, \theta$, etc. have been seen to possess on $A b$ (p. 292). Hence, for all $A b$,

$$
2 \eta=\left(\frac{d r}{d t}\right)^{2}+r^{2}\left(\frac{d \theta}{d t}\right)^{2}+2 \Pi \geqq\left(\frac{d r}{d t}\right)^{2}-\frac{2}{r-1},
$$

and from the preceding inequality,-which is true throughout $A b$-the right-hand member is positive or zero. Thus, $\eta \geqq 0$ throughout $A b$, and all the hypotheses of Theorem 2 are satisfied, which proves our theorem.

TheOREM 4. Let $h$ be any positive constant. Then there exist half orbits extending to infinity with $\eta_{\infty}>h$, and others with $\eta_{\infty}<h$.

We can always take $(d r / d t)_{0}^{2}$ so large that

$$
\left(\frac{d r}{d t}\right)_{0}^{2}-\frac{2}{r_{0}-1}>2 h
$$

the other hypotheses of Theorem 3 being realized. Then $\eta_{\infty}>h$. To prove the second part of the theorem, take

$$
\left(\frac{d r}{d t}\right)_{0}^{2}=\frac{2}{r_{0}-1}
$$

and suppose the other hypotheses of Theorem 3 fulfilled. We may further suppose that $r_{0}$ is so large, and $(d \theta / d t)_{0}$ so small, that

$$
\eta_{0}+\left(\frac{2}{r_{0}-1}\right)^{1 / 2}<h .
$$

This is clearly possible, since the left-hand member is equal to

$$
\frac{1}{r_{0}-1}+\frac{1}{2} r_{0}^{2}\left(\frac{d \theta}{d t}\right)_{0}^{2}+\Pi_{0}+\left(\frac{2}{r_{0}-1}\right)^{1 / 2} \text {. }
$$

Then, I say, this orbit (which extends to infinity, by Theorem 3) will have $\eta_{\infty}<h$. For, as in the proof of Theorem 3,

$$
\left(\frac{d r}{d t}\right)^{2}-\frac{2}{r-1} \geqq 0
$$


If $d r / d t>0$,

$$
d t \leqq\left(\frac{r-1}{2}\right)^{1 / 2} d r .
$$

By $\left(1^{\prime}\right)$, the expression $G=r^{2} d \theta / d t$, and $\left(3^{\prime}\right)$,

$$
d \eta=d G \leqq \frac{d t}{(r-1)^{2}} \leqq \frac{1}{2^{1 / 2}}(r-1)^{-3 / 2} d r .
$$

Integrating from $P_{0}$ to infinity along $A b$,

$$
\eta_{\infty} \leqq \eta_{0}+\left(\frac{2}{r_{0}-1}\right)^{1 / 2}<h .
$$

Similarly for $d r / d t<0$.

COROLlaRy. In Theorem 4, the value of the constant of Jacobi for the half orbits may be chosen arbitrarily.

For it will be seen that we can satisfy our various hypotheses for any given value of $C$.

Note. All the work of this section applies almost verbatim to the motion of a particle $P$ under the Newtonian attraction of arbitrarily distributed masses, animated with an arbitrary continuous motion, provided merely that their total mass be unity, and that they remain within unit distance of the origin. The only alterations are that $\{A b\}$ and $A b$ will not necessarily be analytic, but will have a continuously varying curvature. Naturally, everything about the integral of Jacobi falls out.

3. The character of the orbits in the neighborhood of infinity. We shall consider the half orbit $A b$, with the same notation as before (Fig. 2). The leading theorem here is the following:

THEOREM 5. If $A b$ extends to infinity, it does so with its direction from the origin, the direction of its tangent, and its specific energy all approaching definite limits. In other words, if $\lim _{s \rightarrow \infty} r=\infty$, the remaining conclusions of Theorem 2 are in force.

If at a single point $A^{\prime}$ of $A b$ the hypotheses of Theorem 3 were realized for the curve $A^{\prime} b$, the conclusion of our theorem would follow at once from the latter. We shall assume from now on that such a point $A^{\prime}$ does not occur. Then, whenever $r(s)$ increases with $s$, we shall need to have (after $r>5$ )

$$
\left(\frac{d r}{d t}\right)^{2}<\frac{2}{r-1}
$$


When $r(s)$ decreases ( $s$ increasing) from a maximum at $P_{1}$, this inequality must still be true; otherwise Theorem 3 would deny the existence of $P_{1}$. Thus this inequality will be valid throughout $A b$. Therefore

$$
\lim _{s \rightarrow \infty} \frac{d r}{d t}=0
$$

On substituting the polar expression for $\eta$ in $\left(3^{\prime}\right)$, we have

$$
2 G-\left(\frac{d r}{d t}\right)^{2}-r^{2}\left(\frac{d \theta}{d t}\right)^{2}-2 \Pi=C .
$$

Eliminating $d \theta / d t$ by means of the definition of $G$,

$$
G=r^{2} \frac{d \theta}{d t}
$$

we obtain the quadratic equation in $G$ :

$$
G^{2}-2 r^{2} G+r^{2}\left[2 \Pi+C+\left(\frac{d r}{d t}\right)^{2}\right]=0 .
$$

This determines the two values of $G, G^{\prime}$ and $G^{\prime \prime}$ :

$$
\begin{aligned}
& G^{\prime}=r^{2}+\left(r^{4}-r^{2}\left[2 \Pi+C+\left(\frac{d r}{d t}\right)^{2}\right]\right)^{1 / 2}, \\
& G^{\prime \prime}=r^{2}-\left(r^{4}-r^{2}\left[2 \Pi+C+\left(\frac{d r}{d t}\right)^{2}\right]\right)^{1 / 2}
\end{aligned}
$$

From the equation proved in the paragraph before last, it follows that from a certain point of $A b$ on, $G=G^{\prime \prime}$. Otherwise, by (7), $G \rightarrow \infty$ as $s \rightarrow \infty$. Hence, by ( $\left.3^{\prime}\right), \eta \rightarrow \infty$, and we should be able to apply Theorem 2,-which would give the contradictory result that $\eta$ approaches a limit.

We wish to evaluate $\lim _{s \rightarrow \infty} G$, if it exists. Assume, as we may, that $r$ is large, and expand the expression for $G^{\prime \prime}$ in (7) by means of the binomial theorem. A little computation shows that

hence, by $\left(3^{\prime}\right)$,

$$
\lim _{s \rightarrow \infty} G=\frac{C}{2}
$$

$$
\lim _{s \rightarrow \infty} \eta=\lim _{s \rightarrow \infty} \frac{V^{2}}{2}=0 \text {. }
$$

Now reason with the first equation of $\left(1^{\prime}\right)$ as we did in the proof of Theorem 3, only with the inequality signs reversed. Here we have, since 


$$
G=r^{2} \frac{d \theta}{d t}, \quad \lim _{s \rightarrow \infty} G=\frac{C}{2}
$$

therefore

$$
\begin{aligned}
\frac{d^{2} r}{d t^{2}} & \leqq \frac{G^{2}}{r^{3}}-\frac{1}{(r+1)^{2}} \\
& \leqq \frac{a}{r^{3}}-\frac{1}{(r+1)^{2}} \quad(a, \text { const. }) .
\end{aligned}
$$

Now, by Fig. 2,

$$
-\frac{\partial \Pi}{\partial r} \leqq-F \cos \alpha \leqq-\frac{1}{(r+1)^{2}}\left(1-\frac{1}{r^{2}}\right)^{1 / 2} ;
$$

therefore

$$
\frac{d^{2} r}{d t^{2}} \leqq \frac{a}{r^{3}}-\frac{1}{(r+1)^{2}}\left(1-\frac{1}{r^{2}}\right)^{1 / 2}
$$

Since by hypothesis $A b$ goes to infinity, from a certain point on, the righthand member of this inequality will be negative. But $d^{2} r / d t^{2}<0$ implies that $r$ is a maximum at every apsis. It follows that, after a certain point, $|d r / d t|>0$. The preceding inequality shows, further, that there will be a positive constant $K$ such that, eventually,

$$
\frac{1}{2} \frac{d}{d r}\left(\frac{d r}{d t}\right)^{2}=\frac{d^{2} r}{d t^{2}}<-\frac{K}{(r+1)^{2}} .
$$

Integrating along the orbit, from $(r, \theta)$ to infinity, we get

$$
V^{2} \geqq\left(\frac{d r}{d t}\right)^{2} \geqq \frac{2 K}{r+1}, \quad K>0 .
$$

From this we may pass to the equations (6), $\left(6^{\prime}\right)$, just as we did in the proof of Theorem 2 from the inequality

$$
V^{2} \geqq \frac{2}{r+1},
$$

the only difference being that the result is valid only at a somewhat greater distance from the origin. Thus, all the material is at hand for the proof of the conclusion of Theorem 2, and our theorem is established.

We turn now to some theorems concerning the existence of an asymptote for a half orblt $A b$ reaching to infinity. It has been proved that when $s \rightarrow \infty$, the limits of $V, G$, and $\phi$ exist. Denote them respectively by $V_{\infty}, G_{\infty}$, and $\Phi$. Let homogeneous coördinates $\left(x_{1}, x_{2}, x_{3}\right)$ be introduced in the $X Y$-plane: 


$$
X=\frac{x_{1}}{x_{3}}, \quad Y=\frac{x_{2}}{x_{3}} .
$$

The distance from the origin to the tangent to $A b$ at any point $(r, \theta)$ is clearly

$$
|r \sin \psi|=\left|r^{2} \frac{d \theta}{d s}\right|=\left|\frac{G}{V}\right| .
$$

This tangent makes an angle $\phi$ with the $X$-axis. The use of Hesse's normal form, and an inspection of signs in the various cases, give us the equation of the tangent in the running coördinates $\left(x_{1}, x_{2}, x_{3}\right)$ :

$$
V \sin \phi \cdot x_{1}-V \cos \phi \cdot x_{2}-G \cdot x_{3}=0 .
$$

When $s \rightarrow \infty$, the coefficients approach those of the equation

$$
V_{\infty} \sin \Phi \cdot x_{1}-V_{\infty} \cos \Phi \cdot x_{2}-G_{\infty} \cdot x_{3}=0 \text {. }
$$

This equation becomes illusory when $V_{\infty}=G_{\infty}=0$. But in that case, by $\left(3^{\prime}\right)$, $C=0$, and

$$
\frac{G}{V}=\frac{V}{2}+\frac{\Pi}{V} .
$$

From the next to the last inequality in the proof of Theorem 4, and other previous results, we have

therefore

$$
V|| \geqq\left(\frac{2 K}{r+1}\right)^{1 / 2}, \text { and }|\Pi| \leqq \frac{1}{r-1} ;
$$

$$
\lim _{s \rightarrow \infty} \frac{G}{V}=0 .
$$

Letting $G_{\infty} / V_{\infty}$ stand for this limit, we see that (8) can always be given a meaning. With this interpretation we have

Theorem 6. The half orbit $A b$ approaches the line (8) as asymptote.

We have merely to show that (8) is tangent to $A b$ at a point on the line infinity. There is no loss of generality in assuming that $\Phi=0$. By means of the non-singular projective transformation of the $X Y$-plane into the $X^{\prime} Y^{\prime}$ plane

$$
x_{1}^{\prime}=x_{3}, \quad x_{2}^{\prime}=x_{2}, \quad x_{3}^{\prime}=x_{1},
$$

we carry the line infinity into the $Y^{\prime}$ axis, and (8) into

$$
V_{\infty} \sin \Phi \cdot x_{3}^{\prime}-V_{\infty} \cos \Phi \cdot x_{2}^{\prime}-G_{\infty} \cdot x_{1}^{\prime}=0,
$$


while $A b$ goes into what we shall call $A^{\prime} b^{\prime}$. We wish to show that $\left(8^{\prime}\right)$ is tangent to $A^{\prime} b^{\prime}$ at a point on the $Y^{\prime}$-axis. The equation of the tangent to $A^{\prime} b^{\prime}$ at an arbitrary point is obtained by applying (T) to the equation of the tangent to $A b$ (since projection conserves tangency). Thus we get

$$
V \sin \phi \cdot x_{3}^{\prime}-V \cos \phi \cdot x_{2}^{\prime}-G \cdot x_{1}^{\prime}=0 .
$$

As $s \rightarrow \infty$, its coefficients approach those of $\left(8^{\prime}\right)$. We have merely to interpret this statement in non-homogeneous coördinates to see that this means that $\left(8^{\prime}\right)$ is tangent to $A^{\prime} b^{\prime}$ at a point on the $X^{\prime}$-axis (the origin, as we see from Theorem 4), and our theorem is proved.

Corollary. If $G_{\infty} \neq 0, A b$ approaches an usymptote not passing through the origin when $V_{\infty} \neq 0$, while it is tangent to the line infinity when $V_{\infty}=0$. If $G_{\infty}=0, A b$ always has an asymptote passing through the origin.

All these theorems show how greatly the motion along an orbit reaching to infinity resembles the hyperbolic and parabolic motion in the problem of two bodies. The new feature in the present case is the conceivability of the particle's oscillating about its asymptote. This might be expected, in view of the oscillatory nature of the field of force.

Note. Here again we observe that our work applies verbatim to a much more general class of cases than the restricted problem of three bodies. It applies to those systems mentioned in the note at the close of the last section which possess an integral of Jacobi (3) (where $-\Pi$ is the force function).

\section{Chapter II. Analytic study of infinity}

1. A transformation of the equations of motion. We shall consider the equations of motion in the rotating $x y$-plane. When the variables $x^{\prime}=d x / d t$, $y^{\prime}=d y / d t$ are introduced, the equations I, (2), reduce to a system of the first order:

$$
\begin{aligned}
\frac{d x}{d t} & =x^{\prime}, \\
\frac{d y}{d t} & =y^{\prime}, \\
\frac{d x^{\prime}}{d t} & =2 y^{\prime}+x-\frac{\partial \Pi}{\partial x}, \\
\frac{d y^{\prime}}{d t} & =-2 x^{\prime}+y-\frac{\partial \Pi}{\partial y} .
\end{aligned}
$$


These define in general a unique integral curve through each point of the $x y x^{\prime} y^{\prime}$-space. The same is clearly true of the 3-dimensional manifold composed of the totality of points $\left(x, y, x^{\prime}, y^{\prime}\right)$ corresponding to a given value of the constant of Jacobi, $C$, and determined by equation (3) of Chapter $\mathrm{I}$. In either case. points for which $(x, y)$ is at infinity form a singularity of the integral curves, as we see on examination of (1). It is our object in this section to reduce the singularity to a standard type by means of a change of variables.

We shall perform the transformation

$$
x+(-1)^{1 / 2} y=\frac{1}{\left(\alpha+(-1)^{1 / 2} \beta\right)^{2}},
$$

or

$$
x=\frac{\alpha^{2}-\beta^{2}}{\left(\alpha^{2}+\beta^{2}\right)^{2}}, \quad y=-\frac{2 \alpha \beta}{\left(\alpha^{2}+\beta^{2}\right)^{2}}, \quad x^{2}+y^{2}=\frac{1}{\left(\alpha^{2}+\beta^{2}\right)^{2}} .
$$

After a rather lengthy but straightforward computation, which can be simplified somewhat by the use of the rudiments of the theory of complex variables, equations (2) of Chapter I yield

$$
\begin{aligned}
\frac{d^{2} \alpha}{d t^{2}}= & -\frac{1}{2}\left(\alpha-4 \frac{d \beta}{d t}\right)+\frac{3}{\alpha^{2}+\beta^{2}}\left\{\alpha\left[\left(\frac{d \alpha}{d t}\right)^{2}-\left(\frac{d \beta}{d t}\right)^{2}\right]\right. \\
& \left.+2 \beta \frac{d \alpha}{d t} \frac{d \beta}{d t}\right\}+\frac{1}{4}\left(\alpha^{2}+\beta^{2}\right)^{3} \Pi_{\alpha}, \\
\frac{d^{2} \beta}{d t^{2}}= & -\frac{1}{2}\left(\beta+4 \frac{d \alpha}{d t}\right)+\frac{3}{\alpha^{2}+\beta^{2}}\left\{-\beta\left[\left(\frac{d \alpha}{d t}\right)^{2}-\left(\frac{d \beta}{d t}\right)^{2}\right]\right. \\
& \left.+2 \alpha \frac{d \alpha}{d t} \frac{d \beta}{d t}\right\}+\frac{1}{4}\left(\alpha^{2}+\beta^{2}\right)^{3} \Pi_{\beta},
\end{aligned}
$$

while the integral of Jacobi, I, (3), becomes

$$
\alpha^{2}+\beta^{2}-4\left[\left(\frac{d \alpha}{d t}\right)^{2}+\left(\frac{d \beta}{d t}\right)^{2}\right]=\left(\alpha^{2}+\beta^{2}\right)^{3}(2 \Pi+C) .
$$

On introducing the new variables $\alpha^{\prime}, \beta^{\prime}, \tau$, defined by

$$
\alpha^{\prime}=\frac{d \alpha}{d t}, \quad \beta^{\prime}=\frac{d \beta}{d t}, \quad\left(\alpha^{2}+\beta^{2}\right) d \tau=d t,
$$


the equations may be written in the form

$$
\begin{aligned}
\frac{d \alpha}{d \tau} & =\left(\alpha^{2}+\beta^{2}\right) \alpha^{\prime}, \\
\frac{d \beta}{d \tau} & =\left(\alpha^{2}+\beta^{2}\right) \beta^{\prime}, \\
\frac{d \alpha^{\prime}}{d \tau} & =-\frac{1}{2}\left(\alpha^{2}+\beta^{2}\right)\left(\alpha-4 \beta^{\prime}\right)+3\left\{\alpha\left(\alpha^{\prime 2}-\beta^{\prime 2}\right)+2 \beta \alpha^{\prime} \beta^{\prime}\right\}+\frac{1}{4}\left(\alpha^{2}+\beta^{2}\right)^{4} \frac{\partial \Pi}{\partial \alpha} \\
\frac{d \beta^{\prime}}{d \tau} & =-\frac{1}{2}\left(\alpha^{2}+\beta^{2}\right)\left(\beta+4 \alpha^{\prime}\right)+3\left\{-\beta\left(\alpha^{\prime 2}-\beta^{\prime 2}\right)+2 \alpha \alpha^{\prime} \beta^{\prime}\right\}+\frac{1}{4}\left(\alpha^{2}+\beta^{2}\right)^{4} \frac{\partial \Pi}{\partial \beta}
\end{aligned}
$$

with the integral of Jacobi

$$
\alpha^{2}+\beta^{2}-4\left(\alpha^{\prime 2}+\beta^{\prime 2}\right)=\left(\alpha^{2}+\beta^{2}\right)^{3}(2 \Pi+C) .
$$

The specific energy $\eta$ is likewise found to be

$$
\eta=\frac{\left(2 \alpha^{\prime}+\beta\right)^{2}+\left(2 \beta^{\prime}-\alpha\right)^{2}}{2\left(\alpha^{2}+\beta^{2}\right)^{3}}+\Pi .
$$

It is seen from (2) that the figure in the $x y x^{\prime} y^{\prime}$-space corresponding to the singularity at infinity has gone into the plane $\alpha=\beta=0$ in the $\alpha \beta \alpha^{\prime} \beta^{\prime}$ space. The correspondence between points $\left(x, y, x^{\prime}, y^{\prime}\right)$ and $\left(\alpha, \beta, \alpha^{\prime}, \beta^{\prime}\right)$ is one-to-two, and analytic except when $(\alpha, \beta)$ is at the origin or infinity. Furthermore, the right-hand members of (3) vanish at $\alpha=\beta=0$. Finally, they are analytic in the neighborhood of $(0,0,0,0)$; for

and by (2)

$$
-\Pi=\frac{1-\mu}{r_{1}}+\frac{\mu}{r_{2}},
$$

$$
r_{1}^{-1}=\left[(x-\mu)^{2}+y^{2}\right]^{-1 / 2}=\left(\alpha^{2}+\beta^{2}\right)\left[1-2 \mu\left(\alpha^{2}-\beta^{2}\right)+\mu^{2}\left(\alpha^{2}+\beta^{2}\right)^{2}\right]^{-1 / 2},
$$

which is clearly analytic at $\alpha=\beta=0$. Likewise for $r_{2}^{-1}$. Thus $\partial \Pi / \partial \alpha$ and $\partial I / \partial \beta$ are analytic at $\alpha=\beta=0$, and their power series expansions about that point start with terms of the first degree. This proves our statement.

Thus the singularity we are considering has been reduced to a well known type: a point, at which the right-hand members of the system of differential equations are analytic, but vanish simultaneously. The singularity is isolated in the manifold (4), for the only point of that manifold where $\alpha=\beta=0$ is $(0,0,0,0)$.

The expansions of the right-hand members of (3) at the origin start with terms of the third degree. If the higher terms are removed, we obtain a 
system whose solution is completely known; for it is obtained by setting $\Pi \equiv 0$, and hence corresponds to the motion of the particle $P$ under the action of no forces. In particular, it will admit the integral of energy (5) $(\Pi=0)$.

It would be of great importance to us to know whether the equations (1) or (3) with $I I$ left in will continue to admit a generalization of this integral. Unfortunately, the standard methods for treating such singularities known at the present time do not apply to this case.

It is possible to find a continuous single-valued function which is a generalization of the integral of energy in a region abutting on our singularity, and which is an integral in the sense that it remains constant along the integral curves. Since this investigation leads us to some further information regarding the unstable orbits, we shall undertake it in the following section.

2. The limiting energy as uniform integral. We shall work in the space of the variables $\left(x, y, x^{\prime}, y^{\prime}\right)$, in which the integral curves are determined by the system of equations (1).

Let $\mathfrak{M}$ denote the point set in the real $x y x^{\prime} y^{\prime}$-space, each point of which is characterized by the fact that the unique integral curve of (1) which passes through it* goes to infinity when $t \rightarrow+\infty$. Let $\bar{M}$ be the corresponding point set for $t \rightarrow-\infty$. As everything we shall say for $\mathfrak{M}$ applies, mutatis mutandis, to $\overline{\mathfrak{M}}$, we shall confine ourselves in the sequel to the consideration of $\mathfrak{M}$ and the case $t \rightarrow+\infty$.

Let $\eta_{\infty}=\lim _{t \rightarrow \infty} \eta$. Then $\eta_{\infty}$ is a single-valued function of $\left(x, y, x^{\prime}, y^{\prime}\right)$ on $\mathfrak{M}$; it is obtained by finding the limit of $\eta$ as $t \rightarrow+\infty$ along the integral curve through the point $\left(x, y, x^{\prime}, y^{\prime}\right)$. That $\eta_{\infty}$ exists, and is positive or zero, is a result of the theorems of Chapter $\mathrm{I}$. We shall denote by $\mathfrak{M}^{\prime}$ the subset of $\mathfrak{M}$ for which $\eta_{\infty}>0$.

Let us inquire into the nature of $\mathfrak{M}^{\prime}$. First, it will be seen that if $P$ is a point of $\mathfrak{M}^{\prime}$, there exists a real neighborhood in the $x y x^{\prime} y^{\prime}$-space about $P$ every point of which belongs to $\mathfrak{M}^{\prime}$. From the formula (4) of the preceding chapter, and $G=r^{2} d \theta / d t$,

$$
\left(\frac{d r}{d t}\right)^{2}-\frac{2}{r-1}=-\frac{2}{r-1}-\frac{G^{2}}{r^{2}}-2 \Pi+2 \eta ;
$$

* It is known that the only points through which the integral curve is not unique have $S$ or $J$ (Fig. 1) as their projection in the $x y$-plane. But through such a point there is just one integral curve with a given direction when projected upon this plane, and given value of $C$. So our definition of $\mathfrak{M}$ may be made to go through if we associate a point with a direction in the $x y$-plane, and a value of $C$, whenever the projection of the point in this plane coincides with $S$ or $J$. A similar result is obtained by regularization. In this discussion we assume that the term "integral curve" includes possible equilibrium points. 
since $\lim \eta>0$, it is seen that at a certain point $P_{0}$ of the integral curve through $P$,

$$
\left(\frac{d r}{d t}\right)_{0}^{2}-\frac{2}{r_{0}-1}>0, \quad\left(\frac{d r}{d t}\right)_{0}>0, \quad r_{0}>5
$$

By continuity, there exists a neighborhood $\mathfrak{Y}_{0}$ about $P_{0}$ for every point of which

$$
\left(\frac{d r}{d t}\right)^{2}-\frac{2}{r-1}>0, \frac{d r}{d t}>0, \quad r>5 .
$$

Hence, by Chapter I, Theorem 3, $\mathfrak{A}_{0}$ belongs to $\mathfrak{M}$. But on account of the fact that

$$
2 \eta \geqq\left(\frac{d r}{d t}\right)^{2}-\frac{2}{r-1},
$$

where the right-hand member is positive in $\mathfrak{H}_{0}$ and never decreases as $t \rightarrow+\infty$ along a given integral curve (cf. proof of Chapter I, Theorem 3), it follows that $\mathfrak{A}_{0}$ belongs to $\mathfrak{M}^{\prime}$. If $\mathfrak{A}$ be the neighborhood about $P$ into which $\mathfrak{A}_{0}$ is carried by that deformation of the $x y x^{\prime} y^{\prime}$-space defined by (1) which carries $P_{0}$ into $P$, it is clear that $\mathfrak{A}$ belongs to $\mathfrak{M}^{\prime}$. We leave it to the reader to consider the cases where this deformation may be of an exceptional character.

Further, $\mathfrak{M}^{\prime}$ is a connected point set. Let $P_{1}$ and $P_{2}$ be two points of $\mathfrak{M}^{\prime}$. The integral curves through these points lie in $\mathfrak{M}^{\prime}$. They will, as was seen above, eventually both enter a region in which (6) is satisfied. This latter belongs to $\mathfrak{M}^{\prime}$, and inspection of the formulas reveals that it is connected. Hence, $P_{1}$ and $P_{2}$ may be connected by a curve of points of $\mathfrak{M}^{\prime}$. In conclusion we have

\section{THEOREM 1. The point set $\mathfrak{M}^{\prime}$ is a 4-dimensional continuum.}

Further information about the boundary points of $\mathfrak{M}, \mathfrak{M}^{\prime}, \overline{\mathfrak{M}}$, and $\overline{\mathfrak{M}}^{\prime}$ will be obtained in $\$ 2$ of Chapter IV, in those cases where the problem admits a surface of section.

THEOREM 2. The function $\eta_{\infty}$ is continuous on $\mathfrak{M}^{\prime}$.

From the continuous nature of the deformation defined by (1) in the $x y x^{\prime} y^{\prime}$-space, it will be sufficient to establish the theorem at a point, $P$, in the neighborhood of which the inequalities (6) are verified. For the integral curve through an arbitrary point of $\mathfrak{M}^{\prime}$ eventually enters such a neighborhood. 
Consider the hyperplane perpendicular to the integral curve at $P$. There is a neighborhood, $\mathfrak{B}$, of $P$ in this hyperplane throughout which (6) is satisfied, and within which the angle made by the integral curves with the hyperplane is greater than a certain positive constant. Let $\mathfrak{F}$ be the tubelike 4-dimensional region composed of the halves of those integral curves through $\mathfrak{B}$ along which $t \rightarrow+\infty$. The region $\mathfrak{F}$ clearly reaches to infinity without reëntering its self.

Determine $t$ in $\mathfrak{F}$ so that it is zero upon $\mathfrak{B}$. Then, within this tube $\mathfrak{F}$, $t=\omega\left(x, y, x^{\prime}, y^{\prime}\right)$, where $\omega$ is a single-valued analytic function.

Let the subscript $\left({ }_{0}\right)$ indicate values upon $\mathfrak{B}$. Clearly we have

$$
\eta_{\infty}-\eta_{0}=\int_{0}^{\infty}\left(\frac{d \eta}{d t}\right) d t
$$

where the line integral is taken along the integral curve in $\mathfrak{F}$ issuing from $\left(x_{0}, y_{0}, x_{0}^{\prime}, y_{0}^{\prime}\right)$. If we can show that this integral is a continuous function of $\left(x_{0}, y_{0}, x_{0}^{\prime}, y_{0}^{\prime}\right)$ on $\mathfrak{B}$, the truth of our theorem will follow at once; for, within a neighborhood of $P,\left(x, y, x^{\prime}, y^{\prime}\right)$ are continuous (analytic, in fact) in $\left(x_{0}, y_{0}, x_{0}^{\prime}, y_{0}^{\prime}, t\right)$, and hence in $\left(x_{0}, y_{0}, x_{0}^{\prime}, y_{0}^{\prime}, \omega\left[x, y, x^{\prime}, y^{\prime}\right]\right)$; so that $\left(x_{0}, y_{0}, x_{0}^{\prime}, y_{0}^{\prime}\right)$ may be expressed in terms of such functions of $\left(x, y, x^{\prime}, y^{\prime}\right)$.

Since the integrand is continuous (analytic), we have but to establish the uniform convergence of the integral $\int_{0}^{\infty}(d \eta / d t) d t$ within any closed subregion of $\mathfrak{B}$. Writing

$$
F(t)=F\left(x_{0}, y_{0}, x_{0}^{\prime}, y_{0}^{\prime}, t\right)=\int_{0}^{t}\left(\frac{d \eta}{d t}\right) d t,
$$

we must prove that, if a constant $\epsilon>0$ be assigned, there is a $T$ independent of $\left(x_{0}, y_{0}, x_{0}^{\prime}, y_{0}^{\prime}\right)$ such that $\left|F\left(t_{2}\right)-F\left(t_{1}\right)\right| \leqq \epsilon$ for all $\left|t_{1}\right|>T,\left|t_{2}\right|>T$. We have, to begin with,

Further, by (1), and I, (4),

$$
F\left(t_{2}\right)-F\left(t_{1}\right)=\int_{t_{1}}^{t_{2}}\left(\frac{d \eta}{d t}\right) d t
$$

$$
\frac{d \eta}{d t}=y \frac{\partial \Pi}{\partial x}-x \frac{\partial \Pi}{\partial y}=-\frac{\partial \Pi}{\partial \theta} .
$$

A little computation shows that there exists a positive constant $M$ such that $|\partial \Pi / \partial \theta| \leqq M / r^{2}$ (indeed, $\left.\leqq M / r^{3}\right)$. Further, by Chapter I, formula (4) and $G=r^{2} d \theta / d t$, we have

$$
\lim _{t \rightarrow+\infty} \frac{d r}{d t}=\left(2 \eta_{\infty}\right)^{1 / 2}
$$


the limit being approached from above. Hence, a constant $h>0$ exists such that, within $\mathfrak{F}, d t \leqq h d r$. Hence, if $t_{2} \geqq t_{1}$,

$$
\left|F\left(t_{2}\right)\right|-\left|F\left(t_{1}\right)\right| \leqq M h \int_{r_{2}}^{r_{1}} \frac{d r}{r^{2}}=M h\left(\frac{1}{r_{1}}-\frac{1}{r_{2}}\right),
$$

and this is sufficient to establish the uniform convergence.

THEOREM 3. For any given value of the constant of Jacobi, there exist infinitely many orbits going to infinity with any assigned value of $\eta_{\infty}>0 . \quad A$ similar theorem is true for orbits which come from infinity.

By Chapter I, Theorem 4, there exist orbits going to infinity with $\eta_{\infty}$ smaller than, or greater than, any assigned positive quantity. Since all orbits whose existence is given by that theorem have $\eta_{\infty}>0$, they belong to $\mathfrak{M}^{\prime}$. From the continuity of $\eta_{\infty}$, and the connected nature of $\mathfrak{M}^{\prime}$, our present theorem results.

The existence of orbits going to, or coming from, infinity with $\eta_{\infty}=0$ will be established in Chapter IV, $\S 2$, in the case where there exists a surface of section.

\section{Chapter III. The surface of section}

1. Preliminary remarks. Consider a 3-dimensional manifold, the points of which form a closed set, and which, at each point, is analytic and without singularity. We shall call this the manifold of states of motion, a terminology explained by the fact that we shall think of its points as being in one-to-one correspondence with the states of motion (i.e., position-and-velocity) of a dynamical system. Let there be defined a set of analytic curves in the manifold, just one through each point, and of such a nature that in a suitable neighborhood $\mathfrak{A}$ of each point they appear as the integral curves of the differential system

$$
\frac{d x}{d t}=X(x, y, z), \quad \frac{d y}{d t}=Y(x, y, z), \quad \frac{d z}{d t}=Z(x, y, z),
$$

where $x, y, z$ are Gaussian coördinates figuring in the analytic representation of the manifold of states of motion in $\mathfrak{A} ; X, Y, Z$ are single-valued, analytic, and nowhere all zero within this neighborhood. We shall say that we have a steady flow in the manifold, and speak of the curves as the stream lines, the parameter $t$ as the time. It is understood that this differential system comes to us from the equations of motion of the dynamical system in question; thus the stream lines, and the trajectories of the latter, correspond. We shall admit that there may be a finite number of exceptional points, and a finite number of exceptional curves (which are simple, closed, 
and analytic), at which these properties of the stream lines do not hold. These will be referred to as the singularities of the flow.

By surface of section* we shall mean a connected surface, the points of which form a closed set, having the following properties:

(a) The surface is analytic and without singularities at each of its points.

(b) The boundaries (if any) are either stream lines, or singularities of the flow; and every singularity appears upon the boundary.

(c) Every stream line cuts the surface at least once within every interval of time of length $\tau$, where $\tau$ is the same for all stream lines.

(d) No stream line is tangent to the interior of the surface, and the angle made with the surface by the stream lines either does not approach zero, or else is at most of the first order in the distance from the boundaries.

It is a familiar fact that the restricted problem of three bodies may be divided into various different cases, depending on the form of the ovals of zero velocity, and on the particular region in which the motion takes place. The manifold of states of motion which it is important to consider in the present connection is any one of the connected manifolds of the points $\left(x, y, x^{\prime}, y^{\prime}\right)$ which correspond to a given value of the constant of Jacobi. They are defined analytically by the equation

$$
x^{\prime 2}+y^{\prime 2}=2 \Omega-C
$$

(cf. Chapter I, (3)). In the case where the infinitesimal body moves inside a closed oval containing only one of the massive bodies $S, J$, Birkhoff $\dagger$ has shown that a ring-shaped surface of section exists, provided $\mu$ be taken sufficiently small, and the constant of Jacobi $C>32^{1 / 3}$. It will be shown in this chapter that, with precisely the same restrictions for $\mu$ and $C$, a ring-shaped surface of section exists in the case of motion outside of an oval of zero velocity containing both $S$ and $J$. We shall use the method employed so frequently

* Cf. the developments of this idea in the following:

H. Poincaré, Les Méthodes Nouvelles de la Mécanique Céleste, Paris, Gauthier-Villars, 1899, vol. III, pp. 196-200, 372-381.

H. Poincaré, Sur un théorème de géométrie, Rendiconti del Circolo Matematico di Palermo, vol. 33 (1912), pp. 375-407.

G. D. Birkhoff, The restricted problem of three bodies, Rendiconti del Circolo Matematico di Palermo, vol. 39 (1915), pp. 1-70.

G. D. Birkhoff, Dynamical systems with two degrees of freedom, these Transactions, vol. 18 (1917), pp. $199-300$.

G. D. Birkhoff, Surface transformations and their dynamical applications, Acta Mathematica, vol. 43 (1920), pp. 1-119.

† G. D. Birkhoff, The restricted problem of three bodies, Rendiconti del Circolo Matematico di Palermo, vol. 39 (1915), \$13. 
by Poincaré* and Birkhoff, $\dagger$ which consists in finding an explicit representation of the surface of section in the integrable case $\mu=0$, and generalizing by continuity and analytic continuation of periodic orbits to the case $\mu>0$. It may be remarked that the representation which we shall obtain may be used, with slight modifications, in the case considered by Birkhoff.

2. The surface of section for $\mu=0$. When $\mu=0$, our problem reduces to the problem of two bodies, referred to coördinate axes through the central body which rotate with unit angular velocity in the positive direction. The orbits, therefore, are conic sections, with a focus at the origin, rotating with respect to the $x y$-axes with unit angular velocity in the negative direction.

The manifold of states of motion which we shall consider is given by

$$
v^{2}=r^{2}+\frac{2}{r}-C, \quad r=\left(x^{2}+y^{2}\right)^{1 / 2}, \quad v^{2}=x^{\prime 2}+y^{\prime 2} .
$$

(Cf. Chapter I, (3).) For a given value of $C>3$, the ovals of zero velocity consist of two circles with centers at the origin and radii $r^{\prime}<1$ and $r^{\prime \prime}>1$. Motion is possible within the smaller, or without the larger circle. The latter case is the one we shall consider.

In order to examine the nature of the integral curves in this manifold, we shall transform to a new set of variables, which may be regarded as elements of the orbits. For this purpose it is not possible to use the ordinary elliptic elements, for we are in the presence of parabolic and hyperbolic orbits, for which the elliptic semi-major axis " $a$ " becomes infinite and imaginary, respectively. It will be seen that the variables denoted below by $(e, \vartheta, w)$ are suitable for our purpose.

It will be convenient to introduce the following quantities, together with certain relations between them, familiar in celestial mechanics. Let

$e=$ eccentricity of the orbit;

$\vartheta=$ longitude of the perihelion, measured from the $+x$-axis, and reduced to the interval $0 \leqq \vartheta<2 \pi$;

$w=$ true anomaly of the particle, reduced to the interval $-\pi \leqq w<\pi$;

$$
a= \begin{cases}\text { elliptic semi-major axis } & \text { when } e<1 \\ \text { not defined } & \text { when } e=1 \\ \text { hyperbolic semi-major axis } & \text { when } e>1\end{cases}
$$

* H. Poincaré, Les Méthodes Nouvelles de la Mécanique Céleste, Paris, Gauthier-Villars, 1899, pp. 196-200.

H. Poincare, Sur un theorème de géometrie, Rendiconti del Circolo Matematico di Palermo, vol. 33 (1915), pp. 375-407.

† G. D. Birkhoff, The restricted problem of three bodies, Rendiconti del Circolo Matematico di Palermo, vol. 39 (1915), \$13. 
$\eta$ and $G$ have the same meaning as previously. They are related by Chapter I, $\left(3^{\prime}\right)$ :

$$
2(G-\eta)=C \text {. }
$$

The polar equation of the orbit as given in celestial mechanics is

$$
r=\frac{G^{2}}{1+e \cos w} .
$$

Comparing this with the corresponding equation given in geometry,

$$
r=\frac{ \pm a\left(1-e^{2}\right)}{1+e \cos w}, \quad e>1,
$$

we derive

$$
G^{2}= \begin{cases}+a\left(1-e^{2}\right) & \text { when } e<1 \\ -a\left(1-e^{2}\right) & \text { when } e>1\end{cases}
$$

At perihelion, $d r / d t=0, w=0$, and

also

$$
r=\iota_{0}= \begin{cases}+a(1-e) & \text { when } e<1 \\ -a(1-e) & \text { when } e>1 .\end{cases}
$$

$$
\eta=\frac{1}{2} V^{2}-\frac{1}{r_{0}}=\frac{1}{2} \frac{G^{2}}{r_{0}^{2}}-\frac{1}{r_{0}} .
$$

By the use of these two formulas and of (3) we get

$$
\eta=\left\{\begin{aligned}
-\frac{1}{2 a} & \text { when } e<1 \\
0 & \text { when } e=1 \\
+\frac{1}{2 a} & \text { when } e>1
\end{aligned}\right.
$$

The middle formula follows from the fact that in parabolic motion the velocity $V$, and hence $\eta$, are zero at infinity. Since (4) is a relation between constants, it holds for a general value of $w$. Excluding for the moment the case $e=1$, we get, on eliminating $a$ from (3) and (4):

$$
2 \eta G^{2}=e^{2}-1 \text {. }
$$

Using this to eliminate $G$ from $2(G-\eta)=C$, we obtain

$$
f(\eta) \equiv \eta^{3}+C \eta^{2}+\frac{C^{2}}{4} \eta+\frac{1-e^{2}}{2}=0 .
$$


Although deduced for $e \neq 1$, both these formulas follow at once from (4) when $e=1$.

We are now able to draw some conclusions. Since attention is being restricted to motion outside the circle of radius $r^{\prime \prime}>1$, it is seen that, when $e<1, a \geqq r^{\prime \prime}>1$. Hence, by (4),

$$
\eta \geqq-\frac{1}{2 r^{\prime \prime}}>-\frac{1}{2} \text {. }
$$

From this, from $2(G-\eta)=C$, and from the fact that $C>3$, it follows that

$$
G \geqq \frac{1}{2}\left(C-\frac{1}{r^{\prime \prime}}\right)>1 .
$$

And when $e \geqq 1$, clearly $G>0$. Hence there are no retrograde orbits.*

In order to examine the manner in which $\eta$ depends on $e$ through equation (5), let the curve $\xi=f(\eta)$ be plotted. It is seen that $f^{\prime}(\eta)=0$ has the two roots

$$
\begin{array}{ll}
\eta=-\frac{C}{6}, & \text { when } f\left(-\frac{C}{6}\right)=\frac{1-e^{2}}{2}-\frac{1}{2}\left(\frac{C}{3}\right)^{8} ; \\
\eta=-\frac{C}{2}, & \text { when } f\left(-\frac{C}{2}\right)=\frac{1-e^{2}}{2} .
\end{array}
$$

Further, $f(-\infty)=-\infty$, and $f(+\infty)=+\infty$. Hence the curve has the character shown in Fig. 3.
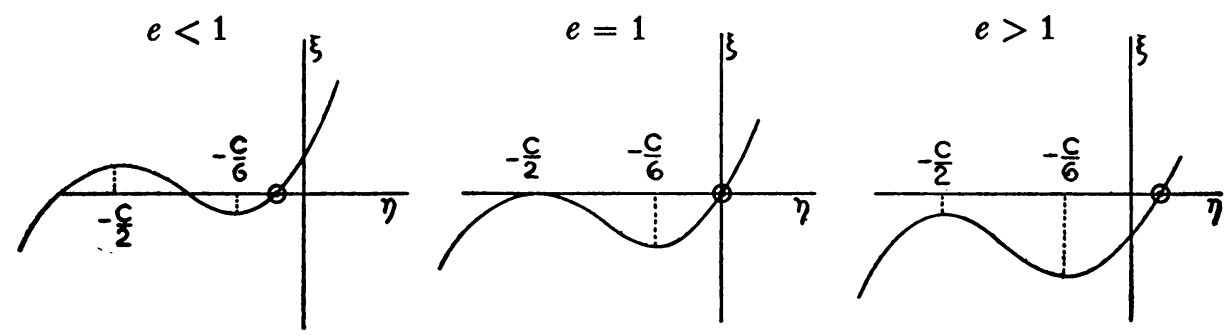

Fig. 3

It follows that for each value of $e$ there is just one value of $\eta$ satisfying both (5) and (6); for by substitution, $f\left(-\frac{1}{2}\right)<0$. Now regard $\eta$ as a function of $e$ defined by (5): $\eta=F(e)$. Differentiating, we get

$$
F^{\prime}(e)=\frac{d \eta}{d e}=\frac{e}{f^{\prime}(\eta)},
$$

* This theorem, which is still true when $\mu>0$ and small, i.e., the case we are going to consider, can be proved for a general value of $\mu$, provided $C$ is not too small. For then it is easy to show by Chapter I, (7), that $G>0$. 
and this is seen from the plot to be positive whenever $e>0$. This and (5) show that as $e$ increases from 0 to $+\infty$, the unique corresponding $\eta=F(e)$ increases from $\eta_{0}>-\frac{1}{2}$ to $+\infty$.

Let us obtain the parametric representation of the manifold of states of motion (1) in the curvilinear coördinates $(e, \vartheta, w)$. Differentiation of (2) along an integral curve gives

$$
\frac{d r}{d t}=\frac{G^{2} e \sin w}{(1+e \cos w)^{2}} \frac{d w}{d t} ;
$$

with this, (2), and $G=r^{2} d \theta / d t$, we can eliminate $d \theta / d t$ and $d r / d t$ from the integral of energy. Use of the formula $2 \eta G^{2}=e^{2}-1$ proved above reduces the result to

$$
\frac{d w}{d t}=\frac{(1+e \cos w)^{2}}{G^{3}} .
$$

Thus, the stream lines in the eqrw-space are determined by the system

$$
\begin{aligned}
& \frac{d e}{d t}=0, \\
& \frac{d \vartheta}{d t}=-1, \\
& \frac{d w}{d t}=\frac{(1+e \cos w)^{2}}{G^{3}},
\end{aligned}
$$

where it is assumed that $G$ is replaced by its value in $e$ from (5):

$$
G=\eta+\frac{C}{2}=F(e)+\frac{C}{2} .
$$

We have but to introduce the polar coördinates $(r, \alpha)$ in the rotating $x y$-plane, when the required uniformizing equations are found to be

$$
\begin{aligned}
r & =\frac{G^{2}}{1+e \cos w}, \\
\alpha & =\vartheta+w, \\
r^{\prime} & =\frac{e \sin w}{G}, \\
\alpha^{\prime} & =-1+\frac{(1+e \cos w)^{2}}{G^{2}},
\end{aligned}
$$

where as before $G=F(e)+C / 2$. 
Through any point $\left(x, y, x^{\prime}, y^{\prime}\right)$, or $\left(r, \alpha, r^{\prime}, \alpha^{\prime}\right)$, of the manifold of states of motion (1) there passes a unique stream line, and hence there corresponds a unique set of values $(e, \vartheta, w)$ in the intervals considered, provided $e>0$. Conversely, it is seen from (8) and (5) that to every set $(e, \vartheta, w)$ such that $1+e \cos w>0, e>0$, there corresponds a unique set of $\left(r, \alpha, r^{\prime}, \alpha^{\prime}\right)$ and of $\left(x, y, x^{\prime}, y^{\prime}\right)$ which are shown to belong to (1).

In order to obtain an adequate representation of the flow, we introduce cylindrical polar coördinates, taking $e$ as radius-vector, $\vartheta$ as angle, and $w$ as altitude. Our manifold of states of motion in the resulting $e \vartheta w$-space appears as the solid of revolution formed by revolving the portion of the half-plane $\vartheta=0$ within $A B c c^{\prime} B^{\prime} A^{\prime}$ (shaded in Fig. 4) about the $w$-axis. This is all seen from the geometric meaning of our variables. The surfaces traced by $B c$ and $B^{\prime} c^{\prime}$ have the equation $1+e \cos w=0$.

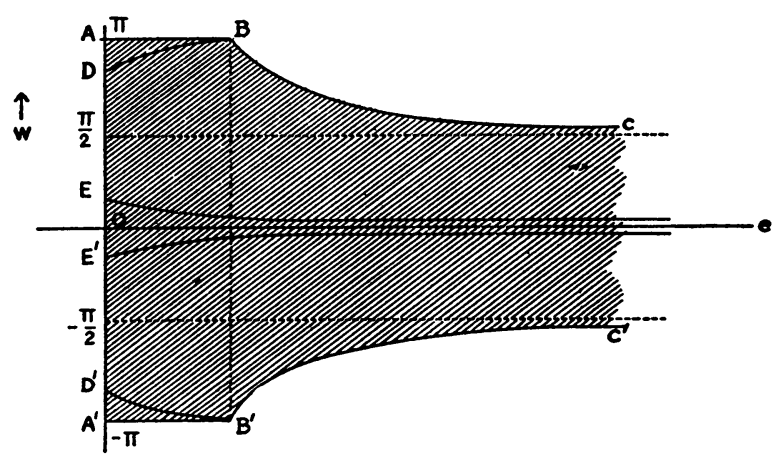

Fig. 4

The single infinitude of states of motion in the circular orbit is ap. propriately represented by the segment $A A^{\prime}$ of the $w$-axis. This leads us to define the variable $w$ on such an orbit: chose an arbitrary point upon it, stationary in the fixed space, and let $w$ be the angle measured from this point to the particle.

We shall now make the convention that all pairs of points $(e, \vartheta,-\pi)$ and $(e, \vartheta, \pi)$ shall be regarded as identical. With this it at once becomes clear from the geometric meaning of the variables that the manifold of states of motion (1) of the $x y x^{\prime} y^{\prime}$-space is in continuous one-to-one correspondence with this open torus-like region of the evrw-space, the singularity at $\infty$ corresponding to the boundary of the torus.

The stream lines in the $e \vartheta w$-space are given by the finite equations

$$
e=e_{0}, \quad \vartheta=-t+\vartheta_{0}, \quad w=w(t) \quad\left[w^{\prime}(t)>0\right] .
$$


For $e_{0}<1$, they are curves on the cylinder $e=e_{0}$, winding steadily upwards, but with variable pitch. They vary from the circular motions $e_{0}=0$ to the parabolic motions $e_{0}=1$. As they approach the latter, the pitch at $w= \pm \pi$ approaches zero. The parabolic motions wind infinitely often about the $w$-axis, approaching the plane $w=\pi$ as $t \rightarrow+\infty$, and $w=-\pi$ as $t \rightarrow-\infty$. The hyperbolic motions are similar in character to the parabolic, only as $t \rightarrow \pm \infty$ they approach the surfaces traced by the revolution of the curves $B c$ and $B^{\prime} c^{\prime}$, corresponding to approach to infinity $(1+e \cos w=0)$.

Consider the portion of the plane $\vartheta=0$ which is shown in the shaded region $A B c A^{\prime} B^{\prime} c^{\prime}$ in Fig. 4. Because of our convention regarding points on $A B$ and $A^{\prime} B^{\prime}$, it is of the nature of a ring, bounded by the circular orbit, and by a closed curve $B c c^{\prime} B^{\prime}$ whose neighborhood corresponds to the neighborhood of infinity in the $x y$-plane. For convenience, we shall refer to these as the "inner" and the "outer" boundaries respectively. It is evident from equations (7) that this ring is cut throughout in the same sense by the stream lines, once by each in the interval of time $2 \pi$. The stream lines are never tangent to the interior of the ring, but make an angle which approaches $\pi / 2$ at the outer boundary, and is of the first order in the distance from the inner boundary. The pitch of these lines approaches the constant value of $-a_{0}^{-3 / 2}\left(a_{0}=\left.a\right|_{e=0}\right)$ at the latter boundary. Thus the transformation of the ring into itself, which the dynamical problem defines, may be extended by continuity to the inner boundary: it advances all points of this a distance of $a_{0}^{-3 / 2}$ upwards. It is clear that the ring is a surface of section for the problem in the evrw-space.

3. The surface of section for $\mu>0$. It was proved by Birkhoff* that the circular orbits in the case $\mu=0$ admit of a unique analytic continuation into symmetric periodic orbits without double points, for a sufficiently small choice of $\mu>0$, provided $C>32^{1 / 3}$. Although stated for motion inside the oval containing one of the massive bodies, it may be seen that the proof holds verbatim for the exterior motion we are considering.

We wish to perform a certain transformation on the $x y x^{\prime} y^{\prime}$-space, in order to carry the manifold of states of motion for $\mu>0$ into that for $\mu=0$. Introduce the polar coördinates $(r, \alpha)$ in the $x y$-plane, and $(v, \beta)$ in the $x^{\prime} y^{\prime}$ plane. Let $r=r^{\prime \prime}$ and $r=r_{0}$ be the equation of the oval of zero velocity and the circular orbit respectively when $\mu=0$; and $r=\Phi(\alpha), r=\Psi(\alpha)$, those of the oval and the analytic continuation when $\mu>0$. Suppose, further, that $\beta=g(\alpha)$ and $\alpha=h(t)$ for the latter. Consider the transformation

* G. D. Birkhoff, The restricted problem of three bodies, Rendiconti del Circolo Matematico di Palermo, vol. 39 (1915), \$11. 


$$
\begin{aligned}
& \bar{r}=[\Psi(\alpha)-\Phi(\alpha)]^{-1} r^{-2}\left\{[\Psi(\alpha)-\Phi(\alpha)] r^{3}\right. \\
&+\left[\Psi(\alpha)^{2}\left\{r_{0}-\Psi(\alpha)\right\}-\Phi(\alpha)^{2}\left\{r^{\prime \prime}-\Phi(\alpha)\right\}\right] r \\
&\left.+\Psi(\alpha) \Phi(\alpha)^{2}\left[r^{\prime \prime}-\Phi(\alpha)\right]-\Phi(\alpha) \Psi(\alpha)^{2}\left[r_{0}-\Psi(\alpha)\right]\right\}, \\
& \bar{\alpha}=\alpha+[A H(\alpha)-\alpha]\left(\frac{r_{0}}{\bar{r}}\right)^{2}, \quad\left(A=\frac{1}{\frac{C}{2}-F(0)}-1 ; \mathrm{cf} .(8)\right), \\
& \bar{v}=v\left(\frac{P}{Q}\right)^{1 / 2}, \\
& \bar{\beta}=\beta+\left(\alpha+\frac{\pi}{2}-g(\alpha)\right)\left(\frac{r_{0}}{\bar{r}}\right)^{2} .
\end{aligned}
$$

Here $H$ is the inverse of $h$; it is single-valued and analytic, since $h$ is, and $d h / d t>0 ; P$ is the quantity $2 \Omega-C$ for $\mu=0$ formed for the point $(\bar{r}, \bar{\alpha})$ (expressed in terms of $(r, \alpha)$ by means of the first two equations of (10)), while $Q$ is the value of $2 \Omega-C$ for the given $\mu>0$ formed for the point $(r, \alpha)$. Since the ovals correspond in virtue of the first two equations of (10), it follows that $P$ and $Q$ are analytically equivalent* on the oval; i.e., $P \equiv Q R$, where $R$ is analytic and distinct from zero at all points of the oval. Hence, $(P / Q)^{1 / 2}$ can be defined in its removable singularities so that it is analytic for all the points considered. Finally, it will be seen that the jacobian of the transformation approaches unity as $r \rightarrow \infty$; and since it is unity when $\mu=0$ ((10) reducing to the identity), and is analytic, we may clearly take $\mu>0$ so small that the jacobian is greater than a certain positive constant throughout the region considered. It is easy to see now that, for $\mu>0$ sufficiently small, (10) has the following properties:

1. It is an analytic correspondence between the manifold of states of motion for $\mu=0$ and that for the given $\mu>0$, varying analytically with $\mu$, and reducing to the identity when $\mu=0$; the dashed letters refer to points of the former.

2. It carries the circular orbit for $\mu=0$ into its analytic continuation for $\mu>0$; and the transformed point describes the circle $\bar{r}=r_{0}$ at the same uniform rate as when $\mu=0$.

3. The displacement which it produces in the points is uniformly bounded, and approaches zero uniformly as $r \rightarrow \infty$.

* Cf. W. F. Osgood, Lehrbuch der Funktionentheorie, Leipzig, B. G. Teubner, 1924, vol. II, pp. $78,87$. 
4. It is symmetric; i.e., if $\left(\bar{x}, \bar{y}, \bar{x}^{\prime}, \bar{y}^{\prime}\right)$ corresponds to $\left(x, y, x^{\prime}, y^{\prime}\right)$, then $\left(\bar{x},-\bar{y},-\bar{x}^{\prime}, \bar{y}^{\prime}\right)$ will correspond to $\left(x,-y,-x^{\prime}, y^{\prime}\right)$.

The last property results from the fact that the equations of motion, Chapter I, (2), are left unchanged when $(x, y, t)$ are replaced by $(x,-y,-t)$, and the consequent symmetric nature of all the elements which enter into (10).

Consider now the transformation of the manifold $v^{2}=2 \Omega-C$ into the solid ring in the $e \vartheta w$-space of Fig. 4 obtained on the successive application of (10) and (8). In order to see what is going on, we shall invert the transformation (8). It is convenient to replace the cylindrical coördinates $(e, \vartheta, w)$ by the rectangular coördinates $(p, q, w): p=e \cos \vartheta, q=e \sin \vartheta$. The equations (8) yield

$$
\begin{aligned}
& p=\cos \alpha \cdot\left(\frac{G^{2}}{r}-1\right)+\sin \alpha \cdot G r^{\prime}=\frac{x}{r}\left(\frac{G^{2}}{r}-1\right)+\frac{y}{r} G r^{\prime}, \\
& q=\sin \alpha \cdot\left(\frac{G^{2}}{r}-1\right)-\cos \alpha \cdot G r^{\prime}=\frac{y}{r}\left(\frac{G^{2}}{r}-1\right)-\frac{x}{r} G r^{\prime}, \\
& w=\arctan \frac{G r^{\prime}}{\frac{G^{2}}{r}-1}, \quad G=r^{2}\left(\alpha^{\prime}+1\right) .
\end{aligned}
$$

It is understood that the points $\left(r, \alpha, r^{\prime}, \alpha^{\prime}\right)$, or $\left(x, y, x^{\prime}, y^{\prime}\right)$, belong to the given manifold of states of motion. These equations are analytic, except for the last when $G^{2}=r$. The periodic orbit $r=\Psi(\alpha)$ is carried into the axis $e=0$, and all the stream lines go into the curves

$$
e=f_{1}(t, \mu), \quad \vartheta=f_{2}(t, \mu), \quad w=f_{3}(t, \mu),
$$

which are analytic in $t$ and $\mu$ for $\mu$ sufficiently small, and reduce to (9) when $\mu=0$. We wish to show that the surface $\vartheta=0$ is related to the stream lines in a manner quite similar to that in the case $\mu=0$ of the last section.

Let us visualise what happens as $\mu$ is increased continuously from zero: the stream lines vary analytically with $\mu$, and will continue to cut the ring in the requisite manner as long as $d \vartheta / d t<$ const. $<0$, and $d w / d t$ remains finite.

The differential equations of motion in the evrw-space may be obtained by differentiating the relations $e=\left(p^{2}+q^{2}\right)^{1 / 2}, \vartheta=\tan ^{-1}(q / p)$, and making use of the equations $\left(8^{\prime}\right)$. We have 


$$
\begin{aligned}
\frac{d e}{d t} & =\cos \vartheta \frac{d p}{d t}+\sin \vartheta \frac{d q}{d t}=\cos (\alpha-\vartheta) \frac{d}{d t}\left(\frac{G^{2}}{r}\right)+\sin (\alpha-\vartheta) \frac{d\left(G r^{\prime}\right)}{d t} \\
\frac{d \vartheta}{d t} & =\frac{1}{e}\left[\cos \vartheta \frac{d q}{d t}-\sin \vartheta \frac{d p}{d t}\right] \\
& =\alpha^{\prime}+\frac{1}{e}\left[\sin (\alpha-\vartheta) \frac{d}{d t}\left(\frac{G^{2}}{r}\right)-\cos (\alpha-\vartheta) \frac{d\left(G r^{\prime}\right)}{d t}\right] \\
\frac{d w}{d t} & =\frac{1}{e}\left[\cos w \frac{d\left(G r^{\prime}\right)}{d t}-\sin w \frac{d}{d t}\left(\frac{G^{2}}{r}\right)\right] .
\end{aligned}
$$

A further use of equations (8) reduces these to

$$
\begin{aligned}
\frac{d e}{d t} & =\cos w \frac{d}{d t}\left(\frac{G^{2}}{r}\right)+\sin w \frac{d\left(G r^{\prime}\right)}{d t}=F_{1}, \\
\frac{d \vartheta}{d t} & =-1+\frac{(1+e \cos w)^{2}}{G^{3}}+\frac{1}{e}\left[\sin w \frac{d}{d t}\left(\frac{G^{2}}{r}\right)-\cos w \frac{d\left(G r^{\prime}\right)}{d t}\right]=F_{2}, \\
\frac{d w}{d t} & =-\frac{1}{e}\left[\sin w \frac{d}{d t}\left(\frac{G^{2}}{r}\right)-\cos w \frac{d\left(G r^{\prime}\right)}{d t}\right]=F_{3} .
\end{aligned}
$$

I say that the $F$ 's are analytic in general, and continuous in all the variables for all the points considered. The only points where trouble might arise are on the axis $e=0$. But if we set $G=r^{2}\left(\alpha^{\prime}+1\right)$, and perform the differentiations, we shall see that the (1/e)-terms all occur in the form $r^{\prime} / e, r^{\prime \prime} / e$, and $\alpha^{\prime \prime} / e$. The first, by equation (8), has the value ( $\left.\sin w\right) / G$. Further, $r^{\prime \prime}$ and $\alpha^{\prime \prime}$ vanish when $e=0$; for then we have uniform circular motion (cf. property 2 of (10)). But $r^{\prime \prime}$ and $\alpha^{\prime \prime}$ are analytic in $\left(r, \alpha, r^{\prime}, \alpha^{\prime}\right.$ ) (cf. the polar equations of motion; this property is not changed by (10)); hence they vanish at least as fast as an expression of the form $K_{1}\left(r-r_{0}\right)+K_{2}\left(\alpha-\alpha_{0}\right)$ $+K_{3}\left(r^{\prime}-r_{0}^{\prime}\right)+K_{4}\left(\alpha^{\prime}-\alpha_{0}^{\prime}\right), K_{i}=$ const., $\left(r_{0}, \alpha_{0}, r_{0}^{\prime}, \alpha_{0}^{\prime}\right)$ being a point on the orbit $e=0$. Here we may assume $K_{2}=0$, since $r^{\prime \prime}=\alpha^{\prime \prime}=0$ when $r=r_{0}$, $r^{\prime}=r_{0}^{\prime}, \alpha^{\prime}=\alpha_{0}^{\prime}, \alpha \neq \alpha_{0}$. And from (8) it appears that $r-r_{0}, r^{\prime}-r_{0}^{\prime}, \alpha^{\prime}-\alpha_{0}^{\prime}$ are of the first order in $e$. Hence $r^{\prime \prime} / e$ and $\alpha^{\prime \prime} / e$ are continuous, and the same is true of the above equations.

When $\mu=0, F_{2}=-1$; also, $F_{2}$ approaches -1 at the outer boundary of the ring, for the corresponding motion approaches two-body motion uniformly at infinity, and by the property 3 , the deformation (10) reduces uniformly to zero as $r \rightarrow \infty$. It follows that $\mu>0$ may be taken so small that $d \vartheta / d t<$ const. $<0$ throughout. Furthermore, $F_{2}$ is everywhere finite when $\mu=0$, and since it is finite in the neighborhood of the outer boundary when $\mu>0$, we may take $\mu>0$ so small that $F_{3}$ is finite throughout. 
The transformation established upon the ring by the stream lines advances points of the inner boundary by an amount which is a continuous function of $\mu$, reducing to $a_{0}^{-3 / 2}$ when $\mu=0$; hence, for a sufficiently small $\mu$, this amount remains greater than a constant, $\omega$. Similarly, the time interval between consecutive intersections is analytic in $\mu$, reducing to $2 \pi$ when $\mu=0$, and at the outer boundary; hence, for a sufficiently small $\mu$, this interval remains less than a constant, $\tau$. Thus we have proved the fundamental theorem:

THEOREM 1. When $C>32^{1 / 3}$, it is possible to take a sufficiently small $\mu_{0}>0$ so that, for each value $0 \leqq \mu \leqq \mu_{0}$, there exists a ring-shaped surface of section for the problem of exterior motion we are considering. The inner boundary is constituted by a closed stream line which corresponds to a symmetric periodic orbit without double points. The outer boundary corresponds to the infinite region in the $x y$-plane. The transformation $T$ established on the ring by the flow advances all points of the inner boundary by an amount greater than a constant $\omega>0$. There are points arbitrarily near to the outer boundary which never make a complete circuit of the ring however often $T$ is applied. Replacing $\left(x, y, x^{\prime}, y^{\prime}\right)$ by $\left(x,-y,-x^{\prime}, y^{\prime}\right)$,-or, what is equivalent, $(e, \vartheta, w)$ by $(e,-\vartheta,-w)$,-carries the ring into itself.

The statement about the rotation in the neighborhood of the outer boundary follows from the existence of half-orbits which reach to infinity without apsides; cf. the interpretation of Fig. 4.

THEOREM 2. Neither of the two boundaries of the image of the ring in the $x y x^{\prime} y^{\prime}$-space can be shrunk continuously to a point without either passing through the other boundary, or departing from the manifold of states of motion $v^{2}=2 \Omega-C$.

It was shown by Birkhoff* that if the variable $\phi=\alpha=\operatorname{arc} \tan \left(y^{\prime} / x^{\prime}\right)$ be introduced, the manifold of states of motion $v^{2}=2 \Omega-C$ in the $x y x^{\prime} y^{\prime}$ space is in one-to-one continuous correspondence with points $(x, y, \phi)$, $0 \leqq \phi<2 \pi$, except that a single point $\left(x_{0}, y_{0}, x_{0}^{\prime}, y_{0}^{\prime}\right)$ on the oval of zero velocity corresponds to the single infinitude of points $\left(x_{0}, y_{0}, \phi\right), 0 \leqq \phi<2 \pi$. This leads us to the following geometric representation. $\dagger$

First, deform the region of motion of the $x y$-plane into the exterior of a circle $K$ in that plane, whose center is at the origin. Let $(x, y)$ correspond to

* G. D. Birkhoff, The restricted problem of three bodies, Rendiconti del Circolo Matematico di Palermo, vol. 39 (1915), p. 21.

$\dagger$ This representation was first applied (incorrectly) by H. Poincaré to the case of motion inside an oval containing one of the massive bodies. Cf. Sur un thêorème de géométrie, Rendiconti del Circolo Matematico di Palermo, vol. 33 (1912), pp. 375-407. 
$(\bar{x}, \bar{y})$ in this deformation. Next, construct all possible circles as follows: (1) the planes of the circles are normal to the $x y$-plane; (2) their centers are in this plane; (3) their two points of intersection with the $x y$-plane are mutual inverts in the circle $K$. Finally, let the point $\left(x, y, x^{\prime}, y^{\prime}\right)$ of the manifold of states of motion be represented by the point upon the circle through $(\bar{x}, \bar{y})$ such that the arc of this circle subtended by $(\bar{x}, \bar{y})$ and the representative point, measured upwards from $(\bar{x}, \bar{y})$, is of angular measure $\phi$.

It is clear that the correspondence is everywhere one-to-one and continuous, and that the points corresponding to the singularity at infinity lie on the $z$-axis in the representative space. This axis clearly threads the periodic orbit, which proves our theorem. We are, of course, using the space of inversion.

We note in passing that the above representation gives an obvious proof of Birkhoff's theorem to the effect that the manifold may be deformed into the space of inversion from which a simple closed curve, corresponding to infinity, has been removed.

4. Two properties of the transformation.* Consider the differential system of the first order, Chapter II, (5), which governs the flow in the $x y x^{\prime} y^{\prime}$-space. The flow is clearly incompressible, i.e., it admits the integral invariant $\iiint \int d x d y d x^{\prime} d y^{\prime}$. For the "equation of continuity" is obviously satisfied. It follows, by reasoning which has been used so often in dynamics that it is unnecessary to repeat it here, that the flow in the manifold of states of motion $v^{2}=2 \Omega-C$ has a positive volume integral invariant, and that the transformation $T$ on the surface of section has a positive area integral invariant. The latter approaches zero at the boundary corresponding to the periodic orbit.

Another property of importance is the following:

The transformation $T$ is the product of two transformations, $R$ and $U$, each of period 2, where $R$ consists in the substitution of $\left(x,-y,-x^{\prime}, y^{\prime}\right)$ for $\left(x, y, x^{\prime}, y^{\prime}\right)$,- or of $(e,-\vartheta,-w)$ for $(e, \vartheta, w)$.

Consider the two points $A$ and $B$ of the surface of section, where $B=T(A)$. By definition of $T, B$ is the next intersection with the surface of the stream line through $A$. Since the equations of motion are unchanged when $x, y$, and $t$ are replaced by $x,-y$, and $-t$, it is seen that the transform of the

- This section is but a paraphrase of the same facts established by Birkhoff for interior motion. Cf. The restricted problem of three bodies, Rendiconti del Circolo Matematico di Palermo, vol. 39 (1915), \$14. 
stream line $\{A B\}$ by $R$ passes through the points $R(A)$ and $R(B)$, but in the opposite order in time. Therefore

$$
R(A)=T R(B)=T R T(A) .
$$

Since $R^{2}=I$, the identity, we have $A=R T R T(A)$, i.e. $R T R T=I$. Hence if we set $U=R T$, we have $T=R U$, and $R^{2}=U^{2}=I$. This fact is true by continuity on the inner boundary.

Chapter IV. The distribution of Periodic AND UnStable motions

1. Symmetric periodic orbits. Throughout this chapter we shall consider the motion of the particle outside the oval of zero velocity containing $S$ and $J$, and shall assume $C$ and $\mu$ to be so restricted that all the results of Chapter III regarding the existence of the surface of section and the transformation $T$ are in force.

In view of the properties of $T$ given by Chapter III, Theorem 1, we have a right to conclude, by Poincarés Geometric Theorem, ${ }^{*}$ that there exist infinitely many periodic orbits for a given value of $C$. But the symmetric properties of the transformation (see Chapter III, \$4) make it possible to arrive at the same result, and at the same time to obtain certain important properties of those of the periodic orbits which are symmetric in the $x$-axis. This state of affairs was taken advantage of by Birkhoff, in the case of interior motion. $\dagger$ The present section is but the most obvious extension of his methods and results to the case of exterior motion we are considering.

We have seen that the differential equations of motion, Chapter I, (2), are unchanged if $(x, y, t)$ be replaced by $(x,-y,-t)$. From this it follows that if we have an orbit which crosses the $x$-axis twice at right angles, this orbit is a symmetric periodic orbit, composed of the portion lying between these two intersections, and its symmetric image in the $x$-axis. Hence no orbit can have more than two such crossings. Further, every periodic orbit which is symmetric in the $x$-axis crosses it at least twice at right angles. For if at $t=t_{1}$ and $t=t_{2}$ we have a pair of successive symmetric crossings at the same point, there will be a right angle crossing when $t=t_{3}=\left(t_{1}+t_{2}\right) / 2$. If $t_{4}$ is the next time the particle crosses at right angles

* H. Poincaré, Sur un théorème de géométrie, Rendiconti del Circolo Matematico di Palermo, vol. 33 (1912), pp. 375-407. 834.

G. D. Birkhoff, Dynamical systems with two degrees of freedom, these Transactions, vol. 18 (1917),

* $†$ G. D. Birkhoff, The restricted problem of three bodies, Rendiconti del Circolo Matematico di Palermo, vol. 39 (1915), \$20. 
at this same point, it will cross at right angles, and at a different point, when $t=\left(t_{3}+t_{4}\right) / 2$. Thus our statement is proved.

Right angle crossings of the $x$-axis may be divided into four classes, according to whether they lie to the right or the left of the oval of zero velocity, and correspond to a relative maximum or minimum distance of the particle from the origin. We shall distinguish

class I, lower apsis at the right of the oval $(\vartheta=0, w=0)$;

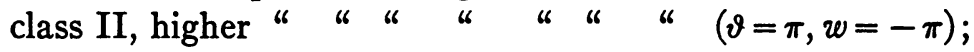

class III, lower " $\quad$ " left $\quad$ " $\quad(\vartheta=\pi, w=0)$;

class IV, higher " $\quad$ " $\quad$ " $\quad$ " $(\vartheta=0, w=-\pi)$.

We have indicated the points in the evw-space corresponding with the states of motion of the respective crossings; the values given are obvious in the case $\mu=0$, and their truth in the general case follows from the symmetric character (property 4) of the transformation (10) of Chapter III.

Consider the ring in the $e \vartheta w$-space (Fig. 4), and the transformation $T$ established upon it by the flow. The lines $O e$ and $A^{\prime} B^{\prime}$ are the loci of the states of motion at crossings of classes I and IV. We have drawn $E e$ and $E^{\prime} e$, the respective loci of the first succeeding and first preceding intersections with the ring, made by those stream lines which pass through points of $\vartheta=\pi, w=0$,-i.e., which have crossings of class III. The lines $D^{\prime} B^{\prime}$ and $D B$ are related in the same way with points of $\vartheta=\pi, w=-\pi$ (crossings of class II). By the symmetry, $E^{\prime} e$ and $D B$ are the reflections of $E e$ and $D^{\prime} B^{\prime}$ in $O e$. When $\mu=0$, the curves have the form indicated, and $O E=A^{\prime} D^{\prime}=\frac{1}{2} a_{0}^{-3 / 2}<\frac{1}{2}$. This continues to be approximately true when $\mu>0$, and small. The essential feature is that $E e$ is always below $B c$, that $D^{\prime} B^{\prime}$ always passes through $B^{\prime}$, and that $O E \geqq \omega>0, A^{\prime} D^{\prime} \geqq \omega$. (Cf. Chapter III, Theorem 1.)

From the nature of the transformation $T$ in the neighborhood of the boundaries, it follows that by repeating it a sufficient number of times it is possible to make the images of $A^{\prime} B^{\prime}, D^{\prime} B^{\prime}, O e, E e$ wind an arbitrary number of times around the ring, and hence yield an arbitrary number of intersections with each of these curves in their initial positions. To such an intersection corresponds an orbit having two right angle crossings of the $x$-axis, of classes depending upon the intersecting curves. We conclude that there exist infinitely many symmetric periodic orbits with crossings of assigned classes.

In order to obtain more detailed information with regard to the periodic orbits, we introduce the characteristic integers $\alpha, \beta$, analogous to the integers employed by Birkhoff for the interior case, but distinct from those. Let $\alpha$ 
be the number of points of intersection that a closed stream line has with the ring; for such an intersection $K, K=T^{\alpha}(K)$; and this equation cannot hold for any number between zero and $\alpha$. Let $\beta$ be the number of times that $K$ circulates around the ring after $\alpha$ applications of $T$. When $\mu=0, \alpha$ is the number of rotations of the ellipse in the $x y$-plane, and $\beta$ the number of revolutions which the particle makes in this ellipse; $\beta / \alpha$ is the mean angular velocity in the fixed space.

THEOREM 1. If $k$ and $l$ are any two integers such that $0<2 \pi l \leqq k \omega<2 \pi(l+1)$, there will exist a symmetric periodic orbit having crossings of the same class, and with $\alpha=2 k, \beta=2 l$, or any equal submultiples of these.

After $k$ applications of $T$, each of the lines $A^{\prime} B^{\prime}, D^{\prime} B^{\prime}, O e, E e$ has its outer boundary point below $B c$, while its point on the inner boundary is advanced by at least $k \omega$. Hence there are on the curve a pair of points, $K$ and $L$, such that $L=T^{k}(K)$, the $k$ images of $K$ having gone around the ring a number of times $l \leqq k \omega /(2 \pi)$. The points $K, L$ will determine a symmetric periodic orbit with $\beta=2 l$ (or a submultiple). It remains to find the corresponding $\alpha$. Since $T=R U$ (Chapter III, \$4), we have

$$
K=T^{-k}(L)=(U R)^{k}(L)=U(R U)^{k-1} R(L),
$$

therefore

$$
R(K)=T^{k}[R(L)]
$$

When $K$ and $L$ are on $A^{\prime} B^{\prime}$ or $O e$,

$$
R(K)=K, \quad R(L)=L \quad\left(\text { for } A^{\prime} B^{\prime} \equiv A B\right),
$$

therefore

$$
K=T^{k}(L) \text {. }
$$

When $K$ and $L$ are on $D^{\prime} B^{\prime}$ or $E e, R(K)$ and $R(L)$ are on $D B$ or $E^{\prime} e$, therefore

$$
R(K)=T^{-1}(K), \quad R(L)=T^{-1}(L),
$$

whence

$$
T^{-1}(K)=T^{k-1}(L), \quad \text { and } \quad K=T^{k}(L),
$$

as before. Hence, in all cases, $K=T^{2 k}(K)$; therefore $\alpha=2 k$ (or a submultiple).

THEOREM 2. If $k$ and $l$ are two integers such that $0<(2 l+1) \pi \leqq k \omega<(2 l+2) \pi$ there will exist a symmetric periodic orbit having crossings of classes I and III, or II and IV, and with $\alpha=2 k+1, \beta=2 l$, or equal submultiples of these. 
After $k$ applications of $T$, the point $E$ advances by at least $k \omega$. Hence the $k$ th image of $E e$ cuts $O e$ in a point $L$, where $L=T^{k}(K), K$ on $E e$, and the $k$ images of $K$ have circulated around the ring $l \leqq k \omega /(2 \pi)-1$ times. This pair of points corresponds to a periodic orbit with crossings of classes $I$ and III, and $\beta=2 l$. To find $\alpha$, we have $R(K)=T^{k}[R(L)]$ as before. Further, $R(K)=T^{-1}(K)$, and $R(L)=L$. Hence $K=T^{k+1}(L)=T^{2 k+1}(K)$, so that $\alpha=2 k+1$. Similar reasoning applies to an orbit having crossings of classes II and IV.

THEOREM 3. If $k$ and $l$ are any integers such that $0<(2 l+1) \pi \leqq k \omega<(2 l+3) \pi$, there will exist a symmetric periodic orbit having crossings of classes I and IV, or II and III, and with $\alpha=2 k, \beta=2 l+1$, or equal submultiples.

After $k$ applications of $T$, the point $A^{\prime}$ advances by at least $k \omega$. If $2 \pi l+\pi \leqq k \omega$, the $k$ th image of $A^{\prime} B^{\prime}$ will cut $O e$ in a point $L, L=T^{k}(K)$, $K$ on $A^{\prime} B^{\prime}$. Here as before, $R(K)=T^{k}[R(L)], R(K)=K, R(L)=L$. Hence $K=T^{k}(L)=T^{2 k}(K)$, and $\alpha=2 k$. Further, it is seen that $\beta=2 l+1$. Similar reasoning is applied in the case of crossings of classes II and III.

THEOREM 4. If $k$ and lare any integers such that $0<(2 l+1) \pi \leqq k \omega<(2 l+3) \pi$, there will exist a symmetric periodic orbit having crossings of classes I and II, or III and IV, and with $\alpha=2 k+1, \beta=2 l+1$, or equal submultiples.

The reasoning is just the same as in Theorem 3, only $D^{\prime} B^{\prime}$ replaces $A^{\prime} B^{\prime}$. Since $K$ is on $D^{\prime} B^{\prime}$, we have $R(K)=T^{-1}(K)$. Hence $K=T^{k+1}(L)$ $=T^{2 k+1}(K)$ and $\alpha=2 k+1$.

2. The distribution of unstable motions. In this section we shall study the manner in which orbits going to infinity, orbits coming from infinity, and orbits which both come from and go to infinity are distributed in the manifold of states of motion. For convenience, we shall consider the representation in the evre-space (Fig. 4), and shall take $\mu$ so small that the transformation $T$ increases the w of each point on the ring by an amount which is positive within the ring, and approaches zero only at $B c c^{\prime} B^{\prime}$. ( $T$ will always have this property near the outer boundary, since there we approach twobody motion. That we may make $T$ behave in this way throughout follows by continuity.)

Consider the surfaces in the manifold of states of motion which are the loci of lower and higher apsides. When $\mu=0$, they are the planes $w=0$ and $w=-\pi$, respectively. For $\mu>0$, they continue to cut the ring in $w=0$ and $w=-\pi$, by the symmetry. Since $\mu$ is small, we are sure that the two surfaces have no point in common. Now two consecutive intersections which a stream line may have with one of them are separated by an intersection with 
the other. Hence, a stream line issuing from a point of the ring has a higher apsis if and only if it has later intersections with the ring above $A B$ (or below $A^{\prime} B^{\prime}$, for $t$ decreasing).

Let $\Lambda$ denote the set of points on the ring characterized by the fact that the stream line issuing from each one has all its later intersections with the ring approaching the boundary $B c$, without approaching $B$ or crossing $A B$. Such a stream line will correspond to a half orbit which goes to infinity without higher apsides as $t \rightarrow+\infty$, and with $\lim _{t \rightarrow+\infty} \eta=\eta_{\infty}>0$. Theorems 3 and 4 of Chapter I allow us to assert that $\Lambda$ is not a null set. We will show that it forms a 2-dimensional continuum upon the ring.

Consider the points of the ring for whose correspondents in the $x y x^{\prime} y^{\prime}$ space the inequalities

$$
\left(\frac{d r}{d t}\right)^{2}-\frac{2}{r-1}>0, \quad \frac{d r}{d t}>0, \quad r>R>5,
$$

are verified. From Chapter I, Theorem 3, and its proof, it follows that they belong to $\Lambda$. When $\mu=0$, they form a band, bounded on one side by the curve $B c$; and, from the nature of Chapter I, (8), (10), they will continue to form such a band for $\mu>0$, provided $R$ be sufficiently large. Now consider any point $P$ of $\Lambda$. After a sufficient number, $k$, of iterations of $T$, its image $T^{k}(P)$ will lie within this band, and consequently will be surrounded by a neighborhood of points of $\Lambda$. On applying $T^{-k}$ to this neighborhood, it is seen that $P$ is surrounded by a neighborhood of points of $\Lambda$. If $Q$ is a second point of $\Lambda$, after a sufficient number, $l$, of repetitions of $T$, the points $T^{l}(P)$ and $T^{l}(Q)$ will both lie in the band, and hence may be connected by a curve of points of $\Lambda$. On applying $T^{-l}$ to this curve, it is seen that $P$ and $Q$ may be connected by such a curve. Thus, $\Lambda$ forms a continuum of points on the ring, and has $B c$ on its boundary.

Consider any boundary point of $\Lambda$, not on $A^{\prime} B^{\prime}$. It is, by definition, a cluster point of points of $\Lambda$, and all its images under $T$ possess this property. Hence the boundary points can never rise above $A B$ under iterations of $T$; otherwise there would be points of $\Lambda$ which rise above this line,-a contradiction. Since $T$ raises every point by an amount which approaches zero only on $B c$ and $B^{\prime} c^{\prime}$, it follows that our boundary points approach $B$, i.e., correspond to half orbits which go to infinity without higher apsides. Furthermore, $\lim _{t \rightarrow+\infty} \eta=0$; otherwise the boundary points would belong to $\Lambda$, and hence be interior points. We say that a boundary point remains such after an application of $T$. It is seen that they form a closed set.

Let $\lambda$ denote the point set defined similarly to $\Lambda$, only with $\lim _{t \rightarrow+\infty} \eta=0$, and with the inclusion of $B$ and $B^{\prime}$. The points of $\lambda$ will all approach $B$ as 
$T$ is iterated indefinitely. Clearly every point of $\lambda$ remains such after $T$. It is easy to see that $\lambda$ is a closed set. We have seen that $\lambda$ contains all those points of the boundary of $\Lambda$ not belonging to $\Lambda$.

As $\lambda$ has infinitely many points on it, it is seen that, with the restrictions under which we are working, we have completed the Theorem 3 of Chapter II, inasmuch as the latter holds for $\eta_{\infty}=0$.

Let $\Lambda^{\prime}$ and $\lambda^{\prime}$ be defined for $t \rightarrow-\infty$ and $T^{-1}$ exactly as $\Lambda$ and $\lambda$ were for $t \rightarrow+\infty$ and $T$. Then everything we have said about the latter point sets holds, mutatis mutandis, for the former. Moreover, on account of the symmetrical character of the transformation $T, \Lambda^{\prime}$ and $\lambda^{\prime}$ are reflections of $\Lambda$ and $\lambda$, respectively, in the line Oe. It follows that the boundaries of $\Lambda$ and $\Lambda^{\prime}$ have at least one point in common, which lies on $O e$. The fundamental theorem here is the following:

TheOREM 5. Either $\Lambda+\lambda$ and $\Lambda^{\prime}+\lambda^{\prime}$ coincide, or else $\Lambda+\lambda$ has points which descend below $A^{\prime} B^{\prime}$ on the application of a suitable power of $T^{-1}$ and $\Lambda^{\prime}+\lambda^{\prime}$ has points which ascend above $A B$ on the application of a suitable power of $T$.

If $\Lambda+\lambda$ and $\Lambda^{\prime}+\lambda^{\prime}$ do not coincide, there will, e.g., be a point in the former which does not belong to the latter. This will descend below $A^{\prime} B^{\prime}$ on the application of a suitable power of $T^{-1}$. By the symmetry, the corresponding property is true with relation to $A B$ and $T$.

When $\mu=0$, the former alternative of Theorem 5 is the one that holds. $\lambda$ and $\lambda^{\prime}$ consist of the line $B B^{\prime}(e=1)$, which is the boundary of both $\Lambda$ and $\Lambda^{\prime}$. In general, as long as $\Lambda+\lambda$ and $\Lambda^{\prime}+\lambda^{\prime}$ coincide, the distribution of unstable motions among the rest is of this simple type.

Unfortunately, I have not succeeded in finding whether $\Lambda+\lambda$ and $\Lambda^{\prime}+\lambda^{\prime}$ continue to coincide when $\mu>0$. It seems extremely probable from a physical point of view that they do not. For consider a very elongated elliptic orbit in the $X Y$-plane $(\mu=0)$. When the particle is at its higher apsis, let $\mu$ take on a positive value. On account of the oscillatory nature of the subsequent force field, the next higher apsis (if any) will in general be distinct from the former. It seems, then, that at least one orbit exists which has a higher apsis, and yet eventually goes to infinity, in one direction at least. Such an orbit would insure the distinctness of $\Lambda+\lambda$ and $\Lambda^{\prime}+\lambda^{\prime}$. We shall, in the rest of this section, pursue the investigation under the hypothesis that these point sets are distinct.

Only Theorem 6 (below) is true without this assumption.

Let $a$ be a region (or connected point set) which belongs to $\Lambda+\lambda$ but not to $\Lambda^{\prime}+\lambda^{\prime}$, and which abuts on $O e$; and let $b$ be its reflection in this line. 
Then $b$ belongs to $\Lambda^{\prime}+\lambda^{\prime}$ but not to $\Lambda+\lambda$, and $a$ and $b$ are separated by points which are in both $\Lambda+\lambda$ and $\Lambda^{\prime}+\lambda^{\prime}$. It is clear that points of $a$ divide their orbits into halves, one of which goes to infinity without higher apsides when $t \rightarrow+\infty$, while the other does not go to infinity, at least without passing through higher apsides, when $t \rightarrow-\infty$. A corresponding property is enjoyed by points of $b$.

Denote by $a_{i}, b_{i}$ the $i$ th images of $a$ and $b$ under $T^{-1}$. Then all the regions $b_{i}$ belong to $\Lambda^{\prime}+\lambda^{\prime}$, but not to $\Lambda+\lambda$. The corresponding property is not true of the $a_{i}$ 's, except perhaps for the first few. For as in the proof of Theorem 5, there will be points of a certain region $a_{\nu}$ which are below $A^{\prime} B^{\prime}$. Suppose that $a_{v}$ is the first $a_{i}$ which has such points, and denote one of the common points of $A^{\prime} B^{\prime}$ and $a_{\nu}$ by $R_{1}^{\prime}$, its reflection in $O e$ by $R_{1}$. Then it is possible to connect $B$ with $R_{1}^{\prime}$ by means of a curve $\left\{B R_{1}^{\prime}\right\}$ of points of $\Lambda+\lambda$.

Since $A B$ and $A^{\prime} B^{\prime}$ are congruent, by our convention with regard to the ring, the regions $a_{i}(i>\nu)$ will come down into the shaded portion of Fig. 4 again through $R_{1} B$. Points of $\Lambda+\lambda$ which have crossed $A B k$ times under iterations of $T^{-1}$ correspond to half orbits which go to infinity as $t \rightarrow+\infty$ after passing through $k$ higher apsides. Hence, points which have crossed $A B$ a different number of times cannot coincide. It follows that there will be points of $\Lambda+\lambda$ (e.g., of a) which go around the ring (crossing $A B$ ) an arbitrary number of times as $T^{-1}$ is indefinitely applied to the point set. Thus, the curve $\left\{B R_{1}^{\prime}\right\}$ of points of $\Lambda+\lambda$ drawn above makes it impossible for those points of $a_{v+1}, a_{v+2}, \cdots$ which go below $A B$ to approach $B^{\prime} c^{\prime}$; hence, by the nature of $T^{-1}$, they will descend, eventually past $A^{\prime} B^{\prime}$ a second time. By recommencing this reasoning arbitrarily often, drawing each time the curves $\left\{R_{1} R_{2}^{\prime}\right\},\left\{R_{2} R_{3}^{\prime}\right\},\left\{R_{3} R_{4}^{\prime}\right\}$, etc., as we formerly drew $\left\{A R_{1}{ }^{\prime}\right\}$, we are able to establish the truth of our statement.

The figures $a_{v+1}, a_{v+2}, \cdots$ form an infinitude of band-like point sets; as we go arbitrarily far out in this sequence, we come upon bands which spiral around the ring an arbitrary number of times. But since the $b$ 's are all above $A^{\prime} B^{\prime}$, it follows that between any two $a$-bands there are bands of points which do not belong either to $\Lambda+\lambda$ or $\Lambda^{\prime}+\lambda^{\prime}$, nor to any of their images under iterations of $T^{-1}$. Points of these separating bands correspond to half orbits which do not go to infinity as $t \rightarrow+\infty$; we do not imply, however, that such half orbits remain within any fixed distance from the origin in the $x y$-plane.

This gives an idea of the complicated nature of the figure which results from $\Lambda+\lambda$ on an indefinite number of applications of $T^{-1}$. If we reflect in $O e$, we obtain the corresponding figure derived from $\Lambda^{\prime}+\lambda^{\prime}$ on an indefinite 
number of applications of $T$. These two figures intersect along $O e$. Thus there are symmetric orbits which both go to and come from infinity, and have an arbitrary number of higher apsides.

By examining the intersections that are possible between the various types of bands of paragraph-before-last and their reflections, it is likewise seen that there are infinitely many orbits which go to infinity but do not come from infinity, and orbits which come from infinity but do not go to infinity. But, since no intersections of unlike bands of the symmetric figures can occur upon $O e$, none of these orbits are symmetric, - a fact which is otherwise obvious.

Consider the infinite succession of ring-shaped regions on the surface of section, contained between the inner boundary and the curves $\left\{R_{i} R_{i+1}^{\prime}\right\}$ and $\left\{R_{i+1}^{\prime} R_{i}^{\prime}\right\}$. As $i \rightarrow \infty$, they approach an inner limiting set, containing at least the inner boundary. This set is carried into itself by $T$. Every point of it is on an orbit which remains within a fixed distance from the origin (independent of the orbit) both for $t \rightarrow+\infty$ and $t \rightarrow-\infty$. (But the converse is not necessarily true.) The set is symmetric in Oe. To points of this set the theory of "Poisson stability" and recurrent motion of Poincaré* and Birkhoff $\dagger$ may be applied.

The inner limiting point set has an area if and only if the periodic orbit constituted by the boundary of the ring is absolutely stable, i.e., of such a nature that, whatever $\epsilon>0$ be assigned, there is a neighborhood of points about this orbit all of whose images under $T^{k}$ and $T^{-k}(k=1,2, \cdots)$ are within $\epsilon$ of the inner boundary.

We shall end this section by a theorem which is entirely independent of the unproved assumption about $\Lambda+\lambda$ and $\Lambda^{\prime}+\lambda^{\prime}$. Birkhoff lays down the following definition: $\ddagger$

"A transformation $T$ will be called transitive if, for any pair of points $P$ and $Q$ on $S$ (the surface) nearby points $P^{\prime}$ and $Q^{\prime}$ respectively can be found such that $Q^{\prime}=T^{n}\left(P^{\prime}\right)$.

"A transformation $T$ is intransitive in the contrary case."

THEOREM 6. The transformation $T$ on our surface of section is intransitive. If $\Lambda+\lambda$ and $\Lambda^{\prime}+\lambda^{\prime}$ coincide, the theorem is obvious, as we see by taking $P$ outside this region, and $Q$ an interior point.

* H. Poincaré, Les Méthodes Nouvelles de la Mécanique Céleste, Paris, Gauthier-Villars, 1899, vol. 3, Chapter 26.

† G. D. Birkhoff, Quelques theorèmes sur le mouvement des systèmes dynamiques, Bulletin de la Société Mathématique de France, vol. 40 (1912), pp. 305-323.

‡ G. D. Birkhoff, Surface transformations and their dynamical applications, Acta Mathematica, vol. 43 (1920), § 61 . 
If $\Lambda+\lambda$ and $\Lambda^{\prime}+\lambda^{\prime}$ do not coincide, take $P$ inside $\Lambda$ and on $O e$. By the symmetry, $P$ will be inside $\Lambda^{\prime}$. Since $P$ is a common point of open regions, it will be surrounded by a neighborhood $U$ of points common to both $\Lambda$ and $\Lambda^{\prime}$. Let $V$ be the region in which the surface of section is cut by the tube of stream lines issuing from a region of the surface $d r / d t=0, d^{2} r / d t^{2}<0$. Since all points of $U$ are on orbits without higher apsides, while those of $V$ are on orbits with higher apsides, it is clear that all the images of the former region under $T^{n}(n= \pm 1, \pm 2, \cdots)$ are distinct from the latter. If we take $Q$ within $V$, our theorem is proved.

3. Some geometrical theorems. In the corresponding treatment of the interior case, Birkhoff* proved the following theorem:

If $\Gamma$ be any closed curve (not a closed stream line) in the manifold of states of motion, there will necessarily exist an infinite number of stream lines which pass through a point of $\Gamma$ twice (or one which does so infinitely many times).

This theorem will still be true in our case under the further hypothesis that the curve in which the stream lines through $\Gamma$ next cut the surface of section contains the inner boundary in its interior. This will insure that this region cuts itself after an application of $T$, a property which would not otherwise necessarily follow in our case, on account of the infinite extent of the surface of section. Apart from this, the proof goes through without modification.

As corollaries we have the following:

(a) infinitely many orbits pass twice through any given point of the $x y$ plane not on the oval of zero velocity;

(b) infinitely many orbits pass twice through a point of the oval of zero velocity;

(c) infinitely many orbits have two higher apsides at an assigned distance from the origin; similarly for lower apsides.

To show that the theorem applies to (a) and (b), the most convenient way is to examine the position of the states of motion in question in the representation used in the proof of Chapter III, Theorem 2. To prove (c), generalize by continuity from the case of $\mu=0$, in the evrw-space.

* G. D. Birkhoff, The restricted problem of three bodies, Rendiconti del Circolo Matematico di Palermo, vol. 39 (1915), §21.

HARVARD UNIVERSITY, Cambridge, Mass. 